\title{
Random-Player Maker-Breaker games
}

\author{
Michael Krivelevich* Gal Kronenberg \\ School of Mathematical Sciences \\ Raymond and Beverly Sackler Faculty of Exact Sciences \\ Tel Aviv University \\ Tel Aviv, 6997801, Israel \\ \{krivelev, galkrone\}@mail.tau.ac.il \\ Submitted: Feb 9, 2015; Accepted: Sep 30, 2015; Published: Oct 16, 2015 \\ Mathematics Subject Classifications: 05C57; 05C80
}

\begin{abstract}
In a $(1: b)$ Maker-Breaker game, one of the central questions is to find the maximal value of $b$ that allows Maker to win the game (that is, the critical bias $b^{*}$ ). Erdős conjectured that the critical bias for many Maker-Breaker games played on the edge set of $K_{n}$ is the same as if both players claim edges randomly. Indeed, in many Maker-Breaker games, "Erdős Paradigm" turned out to be true. Therefore, the next natural question to ask is the (typical) value of the critical bias for MakerBreaker games where only one player claims edges randomly. A random-player Maker-Breaker game is a two-player game, played the same as an ordinary (biased) Maker-Breaker game, except that one player plays according to his best strategy and claims one element in each round, while the other plays randomly and claims $b$ (or $m$ ) elements. In fact, for every (ordinary) Maker-Breaker game, there are two different random-player versions; the $(1: b)$ random-Breaker game and the $(m: 1)$ random-Maker game. We analyze the random-player version of several classical Maker-Breaker games such as the Hamilton cycle game, the perfect-matching game and the $k$-vertex-connectivity game (played on the edge set of $K_{n}$ ). For each of these games we find or estimate the asymptotic values of the bias (either $b$ or $m$ ) that allow each player to typically win the game. In fact, we provide the "smart" player with an explicit winning strategy for the corresponding value of the bias.
\end{abstract}

Keywords: positional games; Maker-Breaker games; random-graphs

\footnotetext{
${ }^{*}$ Supported in part by USA-Israel BSF Grant 2010115 and by grant 912/12 from the Israel Science Foundation.
} 


\section{Introduction}

Let $X$ be a finite set and let $\mathcal{F} \subseteq 2^{X}$ be a family of subsets. In the $(a: b)$ MakerBreaker game $\mathcal{F}$, two players, called Maker and Breaker, take turns in claiming previously unclaimed elements of $X$. The set $X$ is called the board of the game and the members of $\mathcal{F}$ are referred to as the winning sets. Maker claims $a$ board elements per round, whereas Breaker claims $b$ elements. The parameters $a$ and $b$ are called the bias of Maker and of Breaker, respectively. We assume that Breaker moves first. Maker wins the game as soon as he occupies all elements of some winning set. If Maker does not fully occupy any winning set by the time every board element is claimed by either of the players, then Breaker wins the game. We say that the $(a: b)$ game $\mathcal{F}$ is Maker's win if Maker has a strategy that ensures his victory against any strategy of Breaker, otherwise the game is Breaker's win. The most basic case is $a=b=1$, the so-called unbiased game, while for all other choices of $a$ and $b$ the game is called biased.

It is natural to play Maker-Breaker games on the edge set of a graph $G=(V, E)$. In this case, $X=E$ and the winning sets are all edge sets of subgraphs of $G$ which possess some given graph property $\mathcal{P}$. In this case, we refer to this game as the $(a: b)$ $\mathcal{P}$-game. In the special case where $G=K_{n}$ we denote $\mathcal{P}_{n}:=\mathcal{P}\left(K_{n}\right)$. In the connectivity game, Maker wins if and only if his edges contain a spanning tree of $G$. In the perfectmatching game the winning sets are all sets containing $\lfloor|V(G)| / 2\rfloor$ independent edges of $G$. Note that if $|V(G)|$ is odd, then such a matching covers all vertices of $G$ but one. In the Hamiltonicity game the winning sets are all edge sets containing a Hamilton cycle of $G$. In the $k$-connectivity game the winning sets are all edge sets of $k$-vertex-connected spanning subgraphs of $G$.

Playing unbiased Maker-Breaker games on the edge set of $K_{n}$ is frequently in a favor of Maker. For example, it is easy to see (and also follows from [16]) that for every $n \geqslant 4$, Maker can win the unbiased connectivity game in $n-1$ moves (which is clearly also the fastest possible strategy). Other unbiased games played on $E\left(K_{n}\right)$ like the perfectmatching game, the Hamiltonicity game, the $k$-vertex-connectivity game and the $T$-game where $T$ is a given spanning tree with bounded maximum degree, are also known to be easy wins for Maker (see e.g., [7, 9, 12]). It is thus natural to give Breaker more power by allowing him to claim $b>1$ elements in each turn.

Given a monotone increasing graph property $\mathcal{P}$, it is easy to see that the Maker-Breaker game $\mathcal{P}(G)$ is bias monotone. That is, none of the players can be harmed by claiming more elements. Therefore, it makes sense to study $(1: b)$ games and the parameter $b^{*}$ which is the critical bias of the game, that is, $b^{*}$ is the maximal bias $b$ for which Maker wins the corresponding $(1: b)$ game $\mathcal{F}$.

The most fundamental question in the field of Maker-Breaker games is finding the value of the critical bias $b^{*}$. Erdös suggested the following (rather unexpected) approach which has become known as the "probabilistic intuition" or the "Erdős Paradigm". Consider the $(1: b)$ Maker-Breaker game $\mathcal{P}_{n}$ where $\mathcal{P}$ is a monotone graph property. Then according to the intuition, the maximum value of $b$ which allows Maker to win the game playing according to his best strategy should be approximately the same as the maximum value of 
$b$ for which Maker is the typical winner where both players play randomly. Observe that if indeed both players play randomly, then the resulting graph (of Maker) is distributed according to the well-known random graph model $\mathcal{G}(n, m)$ for $m \sim\left(\begin{array}{c}n \\ 2\end{array}\right) /(b+1)$, and thus we can estimate the value of $b^{*}$, according to the Erdös Paradigm, by having the value of the threshold function $m^{*}$ for the same graph property $\mathcal{P}$.

In several important Maker-Breaker games Erdős Paradigm turned out to be true. For example, Chvátal and Erdős [6] showed that for every $\varepsilon>0$, playing with bias $b=\frac{(1+\varepsilon) n}{\ln n}$, Breaker can isolate a vertex in Maker's graph while playing on the board $E\left(K_{n}\right)$. It thus follows that with this bias, Breaker wins every game for which the winning sets consist of subgraphs of $K_{n}$ with positive minimum degree, and therefore, for each such game we have that $b^{*} \leqslant(1+o(1)) n / \ln n$. Much later, Gebauer and Szabó showed in [11] that the critical bias for the connectivity game played on $E\left(K_{n}\right)$ is indeed asymptotically equal to $\frac{n}{\ln n}$. In a relevant development, the first author of this paper proved in [15] that the critical bias for the Hamiltonicity game is asymptotically equal to $\frac{n}{\ln n}$ as well. Indeed, the critical bias in all of the above results (and more) corresponds to the threshold function for the same properties in the random graph model (see e.g., [2]). We refer the reader to $[3,13]$ for more background on the Erdős Paradigm, positional games in general and Maker-Breaker games in particular.

This probabilistic intuition relates the fields of positional games and random graphs. Therefore, it is natural to study Maker-Breaker games that involve randomness. One such version of Maker-Breaker games is the random-turn Maker-Breaker games. A p-randomturn Maker-Breaker game is the same as an ordinary Maker-Breaker game, except that instead of alternating turns, before each turn a biased coin is being tossed and Maker plays this turn with probability $p$ independently of all other turns. Maker-Breaker games under this setting were considered in [17] and in [10].

In this paper we consider a different (randomized) version of Maker-Breaker games. Since the Erdős Paradigm relates biased Maker-Breaker games with biased Maker-Breaker games where both players play randomly, a natural question to ask is how the critical bias changes when only one player plays randomly. In the $(m: b)$ random-player MakerBreaker game one of the players plays according to his best strategy and claims exactly one element in each round, while the other player claims in every round $b$ elements, if he is Brekaer (or $m$ if he is Maker), uniformly among all unclaimed edges.

Clearly, this (random) version of the bias Maker-Breaker games is also bias monotone. More explicitly, if for some bias $b$, it is known that Breaker w.h.p. wins the $(1: b)$ randomBreaker game with respect to some monotone increasing graph property $\mathcal{P}$, then w.h.p. Breaker will also win the $(1:(b+1))$ random-Breaker game (a similar statement holds for the random-Maker game). Therefore, it makes sense to study $(1: b)$ (respectively, $(m: 1))$ games and the parameter $b^{*}$ (respectively, $m^{*}$ ) which is the critical bias of the game, that is, the maximal bias for which the "smart" player w.h.p. (see Section 1.1 for a formal definition of this notion) wins the corresponding random-player game $\mathcal{F}$.

Maker-Breaker games for which one player plays randomly have already been implicitly discussed: Bednarska and Łuczak [4] showed that in the $(1: b)$ (ordinary) Maker-Breaker games on $E\left(K_{n}\right)$, where Maker's goal is to build a copy of a fixed graph $H$, the "random 
strategy" is nearly optimal for Maker. In particular, they proved that if $H$ is a fixed graph with at least 3 non-isolated vertices, then the critical bias for the $H$-game is $b^{*}=$ $\Theta\left(n^{1 / m(H)}\right)$, where $m(H)=\max _{\substack{H^{\prime} \subset H \\ v\left(H^{\prime}\right) \geqslant 3}}\left\{\frac{e\left(H^{\prime}\right)-1}{v\left(H^{\prime}\right)-2}\right\}$. In fact, they showed that if in each turn, Maker claims one element randomly and $b \leqslant c n^{1 / m(H)}$ for some constant $c>0$, then Maker is the typical winner of the game and therefore there exists a deterministic winning strategy for Maker for these values of $b$.

In this paper, we study the critical bias of the random-player version for some wellknown Maker-Breaker games played on the edge set of a complete graph. Furthermore, in cases where the typical winner is "smart", we present strategies that w.h.p. are winning strategies for the game. We do this for both random-Maker and random-Breaker games.

In the $(1: b)$ random-Breaker game, $(X, \mathcal{F})$, there are two players, Maker and Breaker. In each round, Breaker claims $b$ elements from the board, chosen independently uniformly at random among all unclaimed elements, and then Maker claims one element from the board (according to his best strategy). Clearly, the critical bias for the random-Breaker games in bounded from below by the critical bias in the ordinary Maker-Breaker games. We also have a trivial upper bound for the value of $b^{*}$ which is the value of $b$ that, independent of the course of the game, does not allow Maker's graph to achieve the desired property, due to trivial graph-theoretic reasons. For example, in the Hamiltonicity game, Maker needs at least $n$ edges in his graph and thus $b^{*} \leqslant \frac{n}{2}$. We show that in the randomBreaker games, the upper bound is actually the correct value of $b^{*}$ in several well-studied games played on the edge set of $K_{n}$.

Let $\mathcal{H}_{n}$ be the game played on $E\left(K_{n}\right)$ where Maker's goal in to build a Hamilton cycle. The following theorem states that if Breaker is the random player and claims $b \leqslant(1-\varepsilon) \frac{n}{2}$ edges in each round, then Maker can typically win the Hamiltonicity game. Clearly this result is asymptotically tight, since for $b \geqslant(1+\varepsilon) \frac{n}{2}$, after claiming all the edges in the graph, Maker has less than $n$ edges.

Theorem 1. Let $\varepsilon>0$, let $n$ be an integer and let $b \leqslant(1-\varepsilon) \frac{n}{2}$. Then Maker has a strategy which is w.h.p. a winning strategy for the random-Breaker $(1: b) \mathcal{H}_{n}$ game in $(1+o(1)) n$ rounds.

Under the same setting, let $\mathcal{P} \mathcal{M}_{n}$ be the random-Breaker game played on $E\left(K_{n}\right)$ where Maker's goal in to build a graph which contains a perfect matching (or a nearly perfect matching - if $n$ is odd). In the following theorem we prove that if Breaker plays randomly and claims $b \leqslant(1-\varepsilon) n$ edges in each round, then Maker typically wins the perfectmatching game. Clearly this result is also asymptotically tight, since for $b \geqslant(1+\varepsilon) n$, after claiming all edges, Maker has less than $\frac{n}{2}$ edges.

Theorem 2. Let $\varepsilon>0$, let $n$ be an integer and let $b \leqslant(1-\varepsilon) n$. Then Maker has a strategy which is w.h.p. a winning strategy for the $(1: b)$ random-Breaker $\mathcal{P} \mathcal{M}_{n}$ game in $(1+o(1)) \frac{n}{2}$ rounds.

Let $k$ be an integer and let $\mathcal{C}_{n}^{k}$ be the random-Breaker game played on $E\left(K_{n}\right)$ where Maker's goal is to build a $k$-connected graph on $n$ vertices. Using Theorem 1 and Theorem 2, in the following theorem we prove that if Breaker plays randomly and claims 
$b \leqslant(1-\varepsilon) \frac{n}{k}$ edges in each round, then Maker typically wins the $k$-connectivity game. This result is asymptotically tight, since for $b \geqslant(1+\varepsilon) \frac{n}{k}$, after claiming all edges in the graph, the average degree in Maker's graph is smaller than $k$ and therefore the minimum degree is also smaller than $k$.

Theorem 3. Let $\varepsilon>0$, let $n$ be an integer and let $b \leqslant(1-\varepsilon) \frac{n}{k}$. Then Maker has a strategy which is w.h.p. a winning strategy for the $(1: b)$ random-Breaker $\mathcal{C}_{n}^{k}$ game in $(1+o(1)) \frac{k n}{2}$ rounds.

The other version of random-player Maker-Breaker games is the random-Maker games. Unlike the ordinary Maker-Breaker games, for several standard games in this version it turns out to be rather difficult for Maker to win the game. Even in the unbiased version (1: 1), it turns out that in many games Breaker is the typical winner of the game. Therefore, it makes sense to study the $(m: 1)$ random-Maker games and to look for the critical bias of Maker. In the $(m: 1)$ random-Maker games, $(X, \mathcal{F})$, there are two players, Maker and Breaker. In each round, Maker claims $m$ elements from the board, chosen independently uniformly at random among all unclaimed elements, while Breaker claims one element from the board (according to his best strategy). In this case, the critical bias of the game, $m^{*}$, is the maximal value of $m$ for which Breaker is the typical winner of the game.

The first and the most basic game we discuss is the game where Breaker's goal is to isolate a vertex in Maker's graph playing on $E\left(K_{n}\right)$. Recall the result of Chvátal and Erdös [6] about isolating a vertex in biased Maker-Breaker game. In the following theorem we show that playing a random-Maker game on $E\left(K_{n}\right)$, Breaker has a strategy that typically allows him to isolate a vertex in Maker's graph, provided that $m=O(\ln \ln n)$. It thus follows that for this range of $m$, Breaker typically wins every game whose winning sets consist of spanning subgraphs with a positive minimum degree (such as the Hamiltonicity game, the perfect-matching game, the $k$-connectivity game, etc.).

Theorem 4. Let $\varepsilon>0$, let $n$ be an integer and let $m \leqslant\left(\frac{1}{2}-\varepsilon\right) \ln \ln n$. Then w.h.p. Breaker has a strategy to isolate a vertex in Maker's graph while playing the ( $m: 1)$-random-Maker game.

Our next theorem shows that in the random-Maker Hamiltonicity game, if $m=$ $\Omega(\ln \ln n)$ then Maker is the typical winner of the game. Together with Theorem 4, this implies that for the random-Maker Hamiltonicity game, $m^{*}=\Theta(\ln \ln n)$.

Theorem 5. There exists a constant $A>0$ such that if $m \geqslant A \ln \ln n$, then w.h.p. Maker's graph contains a Hamilton cycle while playing the ( $m: 1)$-random-Maker game.

Finally, let $k$ be an integer and consider the $(m: 1)$ random-Maker $k$-vertex-connectivity game played on the edge set of $K_{n}$, where Maker's goal is to build a spanning subgraph which is $k$-vertex-connected. In the following theorem we show that for $m=\Omega_{k}(\ln \ln n)$, Maker is the typical winner of this game. Again, together with Theorem 4 we have that in the random-Maker $k$-connectivity game, $m^{*}=\Theta_{k}(\ln \ln n)$. 
Theorem 6. For every integer $k>0$, there exists a constant $A>0$ such that if $m \geqslant A \ln \ln n$, then playing the ( $m: 1)$-random-Maker game, w.h.p. Maker's graph is $k$-connected.

\subsection{Notation and terminology}

Our graph-theoretic notation is standard and follows that of [18]. In particular we use the following:

For a graph $G$, let $V=V(G)$ and $E=E(G)$ denote its set of vertices and edges, respectively. For subsets $U, W \subseteq V$ we denote by $E_{G}(U, W)$ all the edges $e \in E$ with both endpoints in $U \cup W$ for which $e \cap U \neq \varnothing$ and $e \cap W \neq \varnothing$.

Playing Maker-Breaker game where the board $X$ is the edge set of some graph $G$, we denote by $M$ the subgraph of $G$ consisting of Maker's edges, at any point during the game. Similarly, we denote by $B$ the graph of Breaker and $F=G \backslash(M \cup B)$ is the subgraph of all unclaimed edges, at any point during the game. We say that an edge $e \in E$ is available if $e \in E(F)$.

We also denote by $E_{M}(U, W)$ (respectively, $E_{B}(U, W)$ ) all such edges claimed by Maker (respectively, Breaker) and by $E_{F}(U, W)$ all such unclaimed edges. We let $e(U, W)$ denote the number of edges in $E(U, W)$ (respectively, $e_{M}(U, W), e_{B}(U, W)$ and $e_{F}(U, W)$ are the number of edges in $E_{M}(U, W), E_{B}(U, W)$ and $\left.E_{F}(U, W)\right)$. For a subset $U \subset V$, we write $N_{G}(U)=\{v \in V \backslash U: \exists u \in U$ s.t. $\{u, v\} \in E(G)\}$ and $N_{M}(U)=\{v \in V \backslash U: \exists u \in$ $U$ s.t. $\{u, v\} \in E(M)\}$ (or $\left.N_{B}(U)\right)$.

For a graph $G=(V, E)$ let $\bar{G}=(\bar{V}, \bar{E})$ denote the complement graph of $G$, that is, $\bar{V}=V$ and $\bar{E}=\{\{u, v\} \mid u \neq v \in V,\{v, u\} \notin E\}$. We also write $\Delta(G)$ for the maximum degree in $G$. For a set of vertices $U \subseteq V$, we denote by $G[U]$ the corresponding vertex-induced subgraph of $G$ and we denote by $E_{G}[U]$ the edges of $G[U]$.

We assume that $n$ is large enough where needed. We say that an event holds with high probability (w.h.p.) if its probability tends to one as $n$ tends to infinity. For the sake of simplicity and clarity of presentation, and in order to shorten some of the proofs, no real effort is made to optimize the constants appearing in our results. We also sometimes omit floor and ceiling signs whenever these are not crucial.

\section{Tools}

\subsection{Binomial and Hypergeometric distribution bounds}

We use extensively the following standard bound on the lower and the upper tails of the Binomial distribution due to Chernoff (see, e.g., [1], [14]):

Lemma 7. Let $X \sim \operatorname{Bin}(n, p)$ and $\mu=\mathbb{E}(X)$, then

1. $\operatorname{Pr}(X<(1-a) \mu)<\exp \left(-\frac{a^{2} \mu}{2}\right)$ for every $a>0$.

2. $\operatorname{Pr}(X>(1+a) \mu)<\exp \left(-\frac{a^{2} \mu}{3}\right)$ for every $0<a<1$. 
Let $H G(N, K, n)$ be the Hypergeometric distribution with parameters $N, K$ and $n$, where $N$ is the size of the population containing exactly $K$ successes and $n$ is the number of draws. The following lemma is a Chernoff-type bound on the upper and lower tails of the Hypergeometric distribution.

Lemma 8. Let $N \geqslant 0$, and let $0 \leqslant K, n \leqslant N$ be natural numbers. Let $X \sim H G(N, K, n)$, $\mu=\mathbb{E}[X]=n K N^{-1}$. Then, inequalities 1 and 2 from Lemma 7 hold.

\subsection{Properties of graphs and subgraphs}

First we state a standard fact about subgraphs of large minimum degree. We use this observation in the proof of Theorem 1.

Observation 9. [See, e.g., Ex. 1.3.44 in [18]] Let $r>0$, then every graph with average degree at least $2 r$ contains a subgraph with minimum degree at least $r+1$.

The next two claims are used to prove Theorem 2. In the claims we consider a bipartite graph $G$ satisfying some pseudo-random properties.

Claim 10. Let $0<\varepsilon, \alpha<1$ be constants and let $G=\left(A_{0} \cup A_{1}, E\right)$ be a bipartite graph with parts of size $n$, satisfying the following property: For every $X_{0} \subseteq A_{0}, X_{1} \subseteq A_{1}$ such that $\left|X_{0}\right|=n^{\alpha},\left|X_{1}\right|=n^{\alpha / 2}$, we have $e\left(X_{0}, X_{1}\right) \geqslant \varepsilon\left|X_{0}\right| \cdot\left|X_{1}\right|$.

Then for every two subsets $U_{i} \subseteq A_{i}(i \in\{0,1\}),\left|U_{0}\right|=\left|U_{1}\right|=n^{\alpha}$ the following holds.

(a) The sets of vertices $T_{i}=\left\{v \in U_{i} \mid e\left(v, U_{1-i}\right)<\frac{\varepsilon}{2} n^{\alpha}\right\}$ are of size less than $n^{\alpha / 2}$.

(b) In every set $W_{i} \subseteq A_{i}$ of size $\frac{\varepsilon}{5} n$, there is a vertex $w \in W_{i}$ such that $e\left(w, U_{1-i}\right) \geqslant \frac{\varepsilon}{2} n^{\alpha}$.

Proof. For item $(a)$, if $\left|T_{1}\right| \geqslant n^{\alpha / 2}$, look at the subset $T^{\prime} \subset T_{1},\left|T^{\prime}\right|=n^{\alpha / 2}$. Then, $e\left(T^{\prime}, U_{2}\right) \geqslant \varepsilon\left|T^{\prime}\right| \cdot\left|U_{2}\right|=\varepsilon n^{3 \alpha / 2}$. But, $e\left(T^{\prime}, U_{2}\right)=\sum_{v \in T^{\prime}} e\left(v, U_{2}\right)<\left|T^{\prime}\right| \cdot \frac{\varepsilon}{2} n^{\alpha}=\frac{\varepsilon}{2} n^{3 \alpha / 2}$ - a contradiction. Therefore, $\left|T_{1}\right|<n^{\alpha / 2}$. The proof for $T_{2}$ is similar. Item $(b)$ follows immediately from $(a)$.

In the next claim we show a version of Hall's condition.

Claim 11. Let $\varepsilon>0$ and let $G\left(A_{0} \cup A_{1}, E\right)$ be a bipartite graph with parts of size $n$. Assume that $G$ satisfies the following properties:

1. for every $v \in A_{i}(i \in\{1,2\}), d(v) \geqslant \varepsilon n$, and

2. For every $X_{0} \subseteq A_{0}, X_{1} \subseteq A_{1}$ such that $\left|X_{i}\right|=\varepsilon n$, we have $e\left(X_{0}, X_{1}\right) \geqslant 1$.

Then $G$ contains a perfect matching.

Proof. We need to show that for every $X \subseteq A_{0}$ we have $|N(X)| \geqslant|X|$ (Hall's condition). First, let $X \subseteq A_{0}$ be a set of size $|X| \leqslant \varepsilon n$. Then by item $1,|N(X)| \geqslant|X|$. Next, if $|X|>(1-\varepsilon) n$ then according to item $1,|N(X)|=n$ and we are done. Finally, assume $\varepsilon n<|X| \leqslant(1-\varepsilon) n$ but $|N(X)|<|X|$. But then $|N(X)| \leqslant(1-\varepsilon) n$ and thus for $Z=A_{1} \backslash N(X)$, we have $|Z|>\varepsilon n$ and from item 2 we have $e(X, Z) \geqslant 1$. This is a contradiction. 


\subsection{Expanders}

For positive constants $R$ and $c$, we say that a graph $G=(V, E)$ is an $(R, c)$ - expander if $\left|N_{G}(U)\right| \geqslant c|U|$ holds for every $U \subseteq V$, provided $|U| \leqslant R$. When $c=2$ we sometimes refer to an $(R, 2)$-expander as an $R$-expander. Given a graph $G$, a non-edge $e=\{u, v\}$ of $G$ is called a booster if adding $e$ to $G$ creates a graph $G^{\prime}$ which is Hamiltonian, or contains a path longer than a maximum length path in $G$.

The following lemma states that if $G$ is a "good enough" expander, then it is also a $k$-vertex-connected graph.

Lemma 12. [Lemma $\mathbf{5 . 1}$ from [5]] For every positive integer $k$, if $G=(V, E)$ is an $(R, c)$ - expander with $c \geqslant k$ and $R c \geqslant \frac{1}{2}(|V|+k)$, then $G$ is $k$-vertex-connected.

The next lemma due to Pósa (a proof can be found for example in [2]), shows that every connected and non-Hamiltonian expander has many boosters.

Lemma 13. Let $G=(V, E)$ be a connected and non-Hamilton $R$-expander. Then $G$ has at least $\frac{(R+1)^{2}}{2}$ boosters.

The following standard lemma shows that in expander graphs, the sizes of connected components cannot be too small.

Lemma 14. Let $G=(V, E)$ be an $(R, c)$-expander. Then every connected component of $G$ has size at least $R(c+1)$.

Proof. Assume towards a contradiction that there exists a connected component of size less than $R(c+1)$. Let $V_{0} \subset V$ be the vertex set of this component. Choose an arbitrary subset $U \subseteq V_{0}$ such that $|U|=\min \left\{R,\left|V_{0}\right|\right\}$. Since $G$ is an $(R, c)$-expander and $|U| \leqslant R$, it follows that $\left|N_{G}(U)\right| \geqslant c|U|$. Moreover, note that $N_{G}(U) \subseteq V_{0}$ as $V_{0}$ is a connected component, therefore

$$
\left|V_{0}\right| \geqslant|U|+\left|N_{G}(U)\right| \geqslant|U|+c|U|=(c+1)|U|,
$$

which implies $|U| \leqslant \frac{\left|V_{0}\right|}{c+1}$. On the other hand, since $\left|V_{0}\right|<R(c+1)$ and $|U|=\min \left\{R,\left|V_{0}\right|\right\}$, it follows that $|U|>\frac{\left|V_{0}\right|}{c+1}$, which is clearly a contradiction.

\section{Random Breaker games}

In this section we consider the random-player setting where Breaker plays randomly and in every round claims $b$ elements independently at random, chosen from all available elements. Here we prove Theorems 1, 2 and 3. 


\subsection{Random Breaker Hamiltonicity game}

In this section we prove Theorem 1.

Since this game is bias monotone, we can assume that $b=(1-\varepsilon) \frac{n}{2}$. First we present a strategy for Maker and then prove that during the game he can typically follow this strategy. For this, recall that by $B$ (respectively $M$ ), we denoted Breaker's (or Maker's) graph at any point during the game. We say that some vertex $v$ is free if for every edge $e \in E(M), v \notin e$.

Strategy $\mathbf{S}_{\boldsymbol{H a m}}$ : Maker's strategy is divided into two stages.

Stage I: In this stage Maker's goal in to build a path of length $n-n^{1 / 4}$. For the sake of the argument, Maker thinks of his path at this stage as being directed; the directions will be ignored at later stages. Denote by $R$ the set of vertices that are not in Maker's path.

- Step 1: After Breaker's first move, Maker chooses an available edge $\left\{v_{0}, v_{1}\right\}$ such that $e_{F}\left(\left\{v_{1}\right\}, R\right) \geqslant n^{1 / 5}$. Then Maker updates $P \leftarrow\left\{\vec{v}_{0}, \vec{v}_{1}\right\}$.

In the $k^{\text {th }}$ round $\left(2 \leqslant k \leqslant n-n^{1 / 4}\right)$, after a (random) move by Breaker, Maker acts as follows. For every $w \in V$, let $R_{w}=\{\{w, u\} \in E \mid u \notin P$ and $\{w, u\}$ is available $\}$

- Step $k$ : Let $v$ be the last vertex in the (directed) path $P$. Maker finds a vertex $u \in R_{v}$ such that $\left|R_{u}\right| \geqslant n^{1 / 5}$. Then Maker claims the edge $\{v, u\}$ and updates $P \leftarrow P \cup\{\overrightarrow{v, u}\}$.

The procedure stops when Maker can no longer follow the strategy or after $n-n^{1 / 4}$ times. Following this strategy, at the end of this stage, $\Delta(M) \leqslant 2$.

Stage II: In this stage Maker increases his path vertex by vertex.

- Step 1: Maker looks at the endpoints, $v_{0}, v_{s}$, of his path. If there is an available edge in $E\left(v_{0}, R\right)$ or in $E\left(v_{s}, R\right)$ then Maker claims this edge and repeats Step 1 (after Breaker's move). Otherwise, all of the endpoints of available edges incident to $v_{0}$ and $v_{s}$ are in the path. Let $X_{0}$ and $X_{s}$ be the sets of available edges incident to $v_{0}$ and $v_{s}$, respectively. We split now these sets into 4 sets: $X_{0}=Y_{0} \cup Z_{0}$ and $X_{s}=Y_{s} \cup Z_{s}$ where ||$Y_{0}|-| Z_{0}|| \leqslant 1,|| Y_{s}|-| Z_{s}|| \leqslant 1$ and $Y_{0}$ (respectively, $Y_{s}$ ) are the edges whose other endpoints are closer to $v_{0}$ (respectively, $v_{s}$ ) on the path. In the next 3 turns of Maker, he closes his path to a cycle as follows (see Figure 1 for an illustration).

Case 1: If all endpoints of $Y_{0}$ (other than $v_{0}$ ) come before all endpoints of $Y_{s}$ (other than $v_{s}$ ) in the path, then Maker finds an edge $\left\{v_{0}, v_{i}\right\}$ in $Y_{0}$ and another edge $\left\{v_{j}, v_{s}\right\}$ in $Y_{s}$, such that the edge $\left\{v_{i-1}, v_{j+1}\right\}$ is available and allows him to close his path to a cycle in 3 steps (the vertices of the cycle are the same as the vertices in the path).

Case 2: If all endpoints of $Z_{s}$ (other than $v_{s}$ ) come before all endpoints of $Z_{0}$ (other than $v_{0}$ ) in the path, then Maker chooses an edge $\left\{v_{0}, v_{j}\right\}$ in $Z_{0}$ and another edge $\left\{v_{i}, v_{s}\right\}$ in $Z_{s}$, such that the edge $\left\{v_{i-1}, v_{j+1}\right\}$ is available and allows him to close his 
path to a cycle in 3 steps (the vertices of the cycle are the same as the vertices in the path).

In his next 3 turns, Maker claims the three edges and closes his path to a cycle, as described above. Then, Maker continues to Step 2.

Case 1:
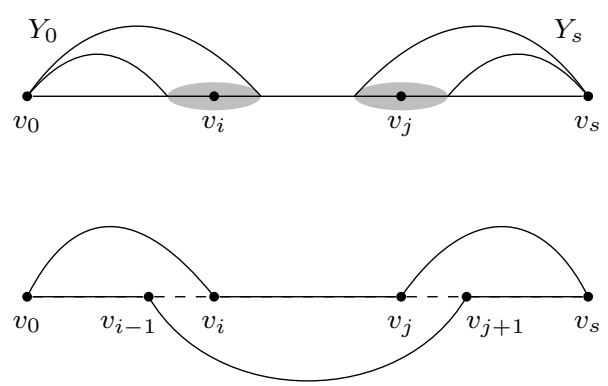

Case 2:
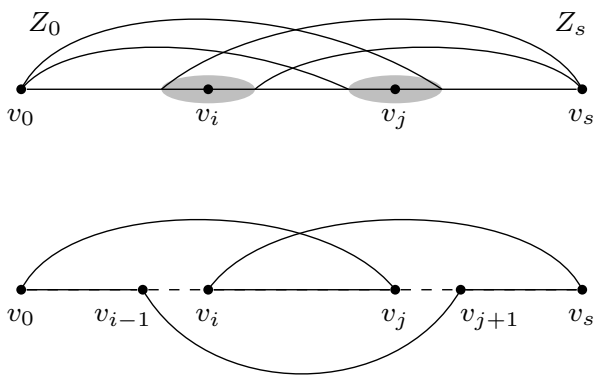

Figure 1: Step 1 in Stage II - Maker claims 3 edges and closes his path to a cycle.

- Step 2: Denote by $C$ the set of vertices in the cycle. Maker claims some available edge $\{c, r\}$, where $c \in C$ and $r \in R$. Denote by $c^{\prime}$ one of the neighbors of $c$ in the cycle. Then Maker updates: $v_{0} \leftarrow c^{\prime}, v_{s-1} \leftarrow c, s \leftarrow s+1$ and $v_{s} \leftarrow r$ and returns to Step 1.

Following this strategy, in every iteration Maker increases his maximum degree by at most 2 and therefore at the end of this stage, $\Delta(M) \leqslant 2 n^{1 / 4}+2$.

Now we prove that w.h.p. Maker can follow this strategy without forfeiting the game. In order to do so, first we need to show that during the game, the degree of Breaker in each vertex is not too large.

Lemma 15. Consider the $(1: b)$ Maker-Breaker game on $E\left(K_{n}\right)$ where Breaker plays randomly and $b=(1-\varepsilon) \frac{n}{2}$. Assume that after $(1+o(1)) n$ rounds $\Delta(M)=O\left(n^{\delta}\right)$ for $a$ constant $0<\delta<1$. Then w.h.p. $\Delta(B) \leqslant\left(1-\frac{\varepsilon}{2}\right) n$.

Proof. Let $v \in V$ and denote by $d_{B}(v)$ the degree of $v$ in Breaker's graph after $(1+o(1)) n$ rounds. Note that after $(1+o(1)) n$ rounds Breaker claimed at most $\left(1-\frac{5}{6} \varepsilon\right)\left(\begin{array}{l}n \\ 2\end{array}\right)$ edges. Also, since the maximal degree in Maker's graph is $O\left(n^{\delta}\right)$ then by the end of the game Maker claimed at most $n^{\delta+1}$ edges. Thus, the number of edges Breaker claimed at each vertex is, essentially, asymptotically stochastically dominated by a hypergeometric random variable with appropriate parameters. Then for $Z \sim H G\left(\left(\begin{array}{l}n \\ 2\end{array}\right)-O\left(n^{\delta+1}\right),\left(1-\frac{5}{6} \varepsilon\right)\left(\begin{array}{l}n \\ 2\end{array}\right), n-1\right)$ and $\mu=\mathbb{E}(Z)$, using Lemma 8 we have,

$$
\begin{aligned}
\operatorname{Pr}\left[d_{B}(v)>\left(1-\frac{\varepsilon}{2}\right) n\right] & \leqslant(1+o(1)) \cdot \operatorname{Pr}\left[Z>\left(1-\frac{\varepsilon}{2}\right) n\right] \\
& \leqslant(1+o(1)) \cdot \operatorname{Pr}\left[Z>\left(1+\frac{\varepsilon}{4}\right) \mu\right] \\
& \leqslant(1+o(1)) e^{-\frac{\varepsilon^{2}}{48} \mu} \leqslant(1+o(1)) e^{-\frac{\varepsilon^{2}}{48}(1-2 \varepsilon) n} .
\end{aligned}
$$


Therefore, by union bound,

$$
\operatorname{Pr}\left[\exists v \in V, \text { s.t. } d_{B}(v)>\left(1-\frac{\varepsilon}{2}\right) n\right] \leqslant n \cdot(1+o(1)) e^{-\frac{\varepsilon^{2}}{48}(1-2 \varepsilon) n}=o(1) .
$$

The next lemma shows that during the game, w.h.p. between any two sets of polynomial size, there are many available edges.

Lemma 16. Consider the $(1: b)$ Maker-Breaker game on $E\left(K_{n}\right)$ where Breaker plays randomly and $b=\Theta(n)$. If at some point during the game $|E(B)| \leqslant\left(1-\frac{5}{6} \varepsilon\right)\left(\begin{array}{l}n \\ 2\end{array}\right)$, then w.h.p. for every constants $0<\beta, \gamma<1$ and for every $X, Y \subseteq V$ such that $|X|=n^{\beta}$, $|Y|=n^{\gamma}$ and $e_{M}(X, Y)=o(|X| \cdot|Y|)$, the number of available elements in $E(X, Y)$ is at least $\frac{\varepsilon}{3}|X| \cdot|Y|$.

Proof. Let $X, Y \subseteq V$ such that $|X|=n^{\beta}$ and $|Y|=n^{\gamma}$, then for $Z \sim H G\left(N,\left(1-\frac{5}{6} \varepsilon\right)\left(\begin{array}{l}n \\ 2\end{array}\right),|X||Y|\right)$ where $N=\left(\begin{array}{c}n \\ 2\end{array}\right)-o\left(n^{2}\right)$ and for $\mu=\mathbb{E}[Z]$,

$$
\begin{aligned}
\operatorname{Pr}\left[e_{B}(X, Y)>\left(1-\frac{\varepsilon}{3}\right)|X| \cdot|Y|\right] & \leqslant(1+o(1)) \operatorname{Pr}\left[Z>\left(1-\frac{\varepsilon}{3}\right)|X| \cdot|Y|\right] \\
& \leqslant\left(1+o(1) \operatorname{Pr}\left[Z>\left(1+\frac{\varepsilon}{4}\right) \mu\right]\right. \\
& \leqslant(1+o(1)) e^{\frac{-\varepsilon^{2}}{48} \mu} \leqslant(1+o(1)) e^{\frac{-\varepsilon^{2}}{48}(1-2 \varepsilon)|X| \cdot|Y|} .
\end{aligned}
$$

Therefore, by the union bound

$$
\begin{aligned}
\operatorname{Pr} & {\left[\exists X, Y,|X|=n^{\beta},|Y|=n^{\gamma}, \text { s.t. } e_{B}(X, Y)>\left(1-\frac{\varepsilon}{3}\right)|X| \cdot|Y|\right] } \\
& \leqslant\left(\begin{array}{c}
n \\
|X|
\end{array}\right)\left(\begin{array}{c}
n \\
|Y|
\end{array}\right) \cdot(1+o(1)) \cdot \exp \left[-\frac{\varepsilon^{2}}{48}(1-2 \varepsilon) n^{\beta+\gamma}\right] \\
& \leqslant(1+o(1))\left(e n^{1-\beta}\right)^{n^{\beta}}\left(e n^{1-\gamma}\right)^{n^{\gamma}} \cdot \exp \left[-\frac{\varepsilon^{2}}{48}(1-2 \varepsilon) n^{\beta+\gamma}\right] \\
& =o(1) .
\end{aligned}
$$

We now prove that w.h.p. Maker can follow his strategy at any point during the game.

\section{Stage I:}

- Step 1: Maker chooses one available edge $\left\{v_{0}, v_{1}\right\}$ and updates $P=\left\{v_{0}, v_{1}\right\}$. From Lemma 15 we know that the number of available edges incident to $v_{1}$ is at least $\frac{\varepsilon}{2} n$. Therefore, in this case clearly $\left|R_{v_{1}}\right| \geqslant n^{1 / 5}$.

- Step $k$ : Let $R$ be the set of vertices that are not in the path $P$. Then $n^{1 / 4} \leqslant|R| \leqslant n$. We now show that if $\left|R_{v}\right| \geqslant n^{1 / 6}$ and $|R| \geqslant n^{1 / 4}$, then there are at least $n^{1 / 8}$ vertices $u \in R_{v}$ such that $\left|R_{u}\right| \geqslant n^{1 / 5}$. Let $T_{v} \subset R_{v}$ be the set of vertices such that for every $u \in T_{v},\left|R_{u}\right| \geqslant n^{1 / 5}$. If $\left|T_{v}\right|<n^{1 / 8}$, then $e_{F}\left(R_{v} \backslash T_{v}, R\right)=\sum_{x \in R_{v} \backslash T_{v}} e_{F}(\{x\}, R) \leqslant$ $\left|R_{v}\right| \cdot n^{1 / 5}$. But according to Lemma 16, w.h.p. $e_{F}\left(R_{v} \backslash T_{v}, R\right) \geqslant \frac{\varepsilon}{3}\left|R_{v} \backslash T_{v}\right| \cdot|R| \geqslant$ 
$\frac{\varepsilon}{4}\left|R_{v}\right| \cdot n^{1 / 4}$. This is clearly a contradiction. Then, Maker chooses one vertex $u \in T_{v}$ and claims $\{v, u\}$.

In the next round, Breaker claims $b$ elements randomly. Let $X$ be the number of edges Breaker claimed from $R_{u}$ in the current round. Then $X \sim H G\left(\Theta\left(n^{2}\right), b,\left|R_{u}\right|\right)$ and $\operatorname{Pr}\left(X>\varepsilon n^{1 / 5}\right)=o\left(\frac{1}{n}\right)$ and therefore after Breaker's move, $\left|R_{u}\right| \geqslant n^{1 / 6}$. Maker repeats this procedure $n-n^{1 / 4}$ times, then by union bound we have that w.h.p. in every step, $\left|R_{u}\right| \geqslant n^{1 / 6}$.

\section{Stage II:}

- Step 1: From Lemma 15 we have that w.h.p. during the game, each vertex has at least $\frac{\varepsilon}{3} n$ available neighbors. Assume that all the endpoints of $X_{0}$ and $X_{s}$ are in the path $P=\left\{v_{0}, v_{1}, \ldots, v_{s}\right\}$.

- Case 1: Let $T_{0}$ be the set of the predecessors of the vertices of $Y_{0}$ along the path (without $v_{0}$ ), and let $T_{s}$ be the set of the predecessors of the vertices of $Y_{s}$ along the path (without $v_{s}$ ). Then w.h.p. $\left|T_{0}\right|,\left|T_{s}\right| \geqslant \frac{\varepsilon}{3} n-1$. Therefore we can use Lemma 16 (note that $E_{M}\left(T_{0}, T_{1}\right)=O\left(n^{1 / 4}\right)$ ) and get that w.h.p. there exists an available edge $\left\{v_{i}, v_{j}\right\}$ in the set $E\left(T_{0}, T_{s}\right)$. In the next 3 turns of Maker, he aims to claim the edges $\left\{v_{i}, v_{j}\right\},\left\{v_{i+1}, v_{0}\right\},\left\{v_{s}, v_{j-1}\right\}$. Then $C=$ $\left\{v_{0}, v_{i+1}, \ldots, v_{j-1}, v_{s}, v_{s-1}, \ldots, v_{j}, v_{i}, v_{i-1}, \ldots, v_{0}\right\}$ would be a cycle of Maker. The probability for Breaker to claim each such edge is $\Theta\left(\frac{b}{n^{2}}\right)$ and therefore (using the union bound over $n^{1 / 4}$ iterations) w.h.p. Maker can achieve his goal.

- Case 2: Let $T_{0}$ be the set of the predecessors of the vertices of $Z_{0}$ along the path (without $v_{s}$ ), and let $T_{s}$ be the set of the predecessors of the vertices of $Z_{s}$ along the path (without $v_{0}$ ). Then, $\left|T_{0}\right|,\left|T_{s}\right| \geqslant \frac{\varepsilon}{3} n-1$. Therefore we can use Lemma 16 and get that there exists an available edge $\left\{v_{i}, v_{j}\right\}$ in the set $E\left(T_{0}, T_{s}\right)$. In the next 3 turns of Maker, he aims to claim the edges $\left\{v_{i}, v_{j}\right\},\left\{v_{i+1}, v_{0}\right\},\left\{v_{s}, v_{j-1}\right\}$. Then $C=\left\{v_{0}, v_{i-1}, \ldots, v_{j+1}, v_{s}, v_{s-1}, \ldots, v_{i}, v_{j}, v_{j-1}, \ldots, v_{0}\right\}$ would be a cycle of Maker. The probability for Breaker to claim each such edge is $\Theta\left(\frac{b}{n^{2}}\right)$ and therefore (using the union bound over $n^{1 / 4}$ iterations) w.h.p. Maker can achieve his goal.

- Step 2: From Lemma 15, we have that w.h.p. for every $v \in R, d_{F}(v) \geqslant \frac{\varepsilon}{3} n$. Since $|R|=O\left(n^{1 / 4}\right)$ it follows that for some (in fact for any) $v \in R$, w.h.p. there exists a vertex $u \in C$ such that $\{u, v\}$ is available.

\subsection{Random Breaker perfect-matching game}

In order to prove Theorem 2, we will use Maker's strategy in the perfect-matching game played on $E\left(K_{n, n}\right)$. Therefore, we now analyze the random-Breaker perfect-matching game where the board of the game is $E\left(K_{n, n}\right)$. Since this game is bias monotone, we can assume that $b=(1-\varepsilon) n$. We prove the following theorem: 
Theorem 17. Let $\varepsilon>0$, let $n$ be a sufficiently large integer and let $b=(1-\varepsilon) n$. Then Maker has a strategy which is w.h.p. a winning strategy for the $(1: b)$ random-Breaker perfect-matching game played on $E\left(K_{n, n}\right)$ in $(1+o(1)) n$ rounds.

Note that Theorem 2 follows easily from Theorem 17. Indeed, at the beginning of the game Maker focuses on two disjoint subsets of $V\left(K_{n}\right)$, each of size $\left\lfloor\frac{n}{2}\right\rfloor$, and pretends to play the perfect-matching game on $E\left(K_{\left\lfloor\frac{n}{2}\right\rfloor,\left\lfloor\frac{n}{2}\right\rfloor}\right)$ (Maker plays only on the edges between the two sets and ignores all other edges). Then, in every round, w.h.p. at least $\left(1-\frac{3 \varepsilon}{2}\right)\left\lfloor\frac{n}{2}\right\rfloor$ of Breaker's edges will be contained in one of the two sets and therefore his bias in the simulated game is actually at most $\left(1-\frac{\varepsilon}{2}\right)\left\lfloor\frac{n}{2}\right\rfloor$.

Proof of Theorem 17 First we present a strategy for Maker and then prove that w.h.p. during the game he can follow this strategy.

Strategy $\mathbf{S}_{\boldsymbol{P M}}$ : Maker's strategy is divided into three stages. Denote by $B$ (or $M$ ), Breaker's (or Maker's) graph at any point during the game. We also write $G=M \cup B$.

Stage I: In this stage Maker's goal is to claim a matching $P$ of size $n-n^{\alpha}$ where $0<\alpha<\frac{1}{3}$ is a constant to be chosen later. Denote by $R$ the set of vertices not touched by any edge of $M$ and denote $E[R]=\left\{e \in E\left(K_{n, n}\right)|| e \cap R \mid=2\right\}$. In every turn, Maker chooses two vertices $x, y \in R$ and claims the edge $\{x, y\}$. Maker repeats this procedure $n-n^{\alpha}$ times and then moves to the next stage. Note that at any point in this stage $\Delta(M) \leqslant 1$. If at some point during this stage Maker cannot follow this strategy, then he forfeits the game. This stage takes $n-n^{\alpha}$ rounds.

Stage II: In this stage, Maker "fixes" his graph by replacing some of the edges in the subgraph $P$. Denote again by $R$ the set of vertices such that $R \cap V\left(M_{1}\right)=\varnothing$ where $M_{1}$ is Maker's graph from Stage I and let $P^{\prime} \leftarrow P$. Let $T=\left\{v \in R \mid e_{F}(v, R)<\frac{\varepsilon}{4} n^{\alpha}\right\}$. For every $v \in T$, Maker chooses an edge $e=\{u, w\} \in P$ such that the edge $\{v, u\}$ is available, $e_{F}(w, R) \geqslant \frac{\varepsilon}{8} n^{\alpha}$, and then claims the edge $e^{\prime}=\{v, u\}$ and updates $P \leftarrow P \backslash\{e\}$, $P^{\prime} \leftarrow\left(P^{\prime} \cup\left\{e^{\prime}\right\}\right) \backslash\{e\}$ and $R \leftarrow(R \backslash\{v\}) \cup\{w\}$. Maker repeats this procedure $|T|$ times. If at some point during this stage Maker cannot do so, then he forfeits the game. Denote Maker's new graph by $M_{2}$. Observe that by the end of this stage, $P^{\prime}$ is a matching of size $n-n^{\alpha}$ and that $|R|=2 n^{\alpha}$. Furthermore, $\Delta\left(M_{2}\right) \leqslant 3$. We will show later that this stage takes at most $\Theta\left(n^{\alpha}\right)$ rounds and that every vertex $v \in R$ satisfies $e_{F}(v, R) \geqslant \frac{\varepsilon}{5} n^{\alpha}$ by the end of this stage.

Stage III: Let $R$ the set of vertices from Stage II. Note that $|R|=2 n^{\alpha}$. In this stage, Maker plays only on the graph $G^{\prime}=K_{n, n}[R]=\left(U_{1} \cup U_{2}, E^{\prime}\right)$, note that $\left|U_{1}\right|=\left|U_{2}\right|=n^{\alpha}$. At the beginning of this stage, Maker finds a set of available edges $P_{1}$ such that $P_{1}$ is a perfect matching in $G^{\prime}$. In each turn, Maker claims an unclaimed edge $e=\{u, v\}$ from $P_{1}$. Maker repeats this procedure $n^{\alpha}$ times. If at some point during this stage Maker cannot do so, then he forfeits the game. Observe that during this stage, the degree of the vertices that are not in $V\left(G^{\prime}\right)=R$ stays the same as in Stage II, and the degree of the vertices in $G^{\prime}$ increases by one. Therefore, by the end of this stage $\Delta(M)=O(1)$.

It is evident that $P^{\prime} \cup P_{1}$ is a perfect matching and therefore following the suggested strategy, w.h.p. Maker wins the game in $n-n^{\alpha}+\Theta\left(n^{\alpha}\right)+n^{\alpha}=n(1+o(1))$ rounds. It remains to prove that w.h.p. Maker can follow this strategy without forfeiting the game. To this end, we first state two lemmas concerning Breaker's graph by the end of the game. 
The proofs of the lemmas are essentially identical to the proofs of Lemma 16 and Lemma 15 , respectively.

Lemma 18. Consider the $(1: b)$ Maker-Breaker game on $E\left(K_{n, n}\right)$ (the bipartite complete graph with sides $\left.V_{1}, V_{2}\right)$, where Breaker plays randomly and $b=\Theta(n)$. If at some point of the game $|E(B)| \leqslant\left(1-\frac{5}{6} \varepsilon\right) n^{2}$ and $\Delta(M)=O(1)$, then w.h.p. for every constants $0<\beta, \gamma<1$ and for every $X_{1} \subseteq V_{1}, X_{2} \subseteq V_{2}$ such that $\left|X_{1}\right|=n^{\beta},\left|X_{2}\right|=n^{\gamma}$, the number of available elements in $E\left(X_{1}, X_{2}\right)$ is at least $\frac{\varepsilon}{3}\left|X_{1}\right| \cdot\left|X_{2}\right|$.

Corollary 19. Consider the $(1: b)$ Maker-Breaker game on $E\left(K_{n, n}\right)$ where Breaker plays randomly and $b=(1-\varepsilon) n$. If $\Delta(M)=O(1)$, then after $(1+o(1)) n$ rounds w.h.p. between every two sets of vertices $X_{i} \subset V_{i}(i \in\{1,2\})$ such that $\left|X_{i}\right| \geqslant n^{\alpha}$, there exists an unclaimed edge.

The next lemma shows that during the game, the degree in Breaker's graph is not too close to $n$.

Lemma 20. Consider the $(1: b)$ Maker-Breaker game on $E\left(K_{n, n}\right)$ where Breaker plays randomly and $b=(1-\varepsilon) n$. If $\Delta(M)=O(1)$, then after $(1+o(1)) n$ rounds, w.h.p. $\Delta(B) \leqslant\left(1-\frac{\varepsilon}{2}\right) n$.

Now we are ready to prove that w.h.p. Maker can follow his strategy in every stage.

Stage I: We need to prove that at any point during this stage Maker can find unclaimed edge $\{x, y\}$ such that $\{x, y\} \cap V(M)=\varnothing$. Indeed, at any point during this stage we have $|R| \geqslant 2 n^{\alpha}$, and by Corollary 19 we know that there is an unclaimed edge in $R$. Therefore, Maker can claim the desired edge.

Stage II: First we show that the number of vertices $v \in R$ such that $e_{F}(v, R)<\frac{\varepsilon}{8} n^{\alpha}$ is small.

Using Lemma 18 for $R$, w.h.p. the graph $F$, by the end of the game, satisfies the property from Claim 10, and thus, w.h.p. $|T| \leqslant 2 n^{\alpha / 2}$. We write $T_{0}=V_{0} \cap T$ and $T_{1}=V_{1} \cap T$ where $V_{0}$ and $V_{1}$ are the parts of $K_{n, n}$. Then $\left|T_{1}\right|,\left|T_{0}\right| \leqslant n^{\alpha / 2}$.

In order to replace the bad vertices in $R$ (that is, the vertices of $T$ ), note that for every vertex $v \in T_{i}$ the number of candidates to replace $v$ is large. Indeed, from Lemma 20, w.h.p. the minimum degree in $F$ is at least $\frac{\varepsilon}{4} n$, therefore w.h.p. for every $v \in T_{i}, e_{F}\left(v, V_{1-i} \backslash\right.$ $(R \cup T)) \geqslant \frac{\varepsilon}{4} n-2 n^{\alpha}-2 n^{\alpha / 2} \geqslant \frac{\varepsilon}{5} n$. For every $v \in T_{i}$ denote $W_{v}=\left\{w \in V_{i} \backslash(T \cup\right.$ $R) \mid \exists u,\{w, u\} \in P,\{v, u\} \in F\}$, so $\left|W_{v}\right| \geqslant \frac{\varepsilon}{5} n>n^{\alpha}$. Since w.h.p. the graph $F$ satisfies the property from Claim 10, we have that w.h.p. there exists $w \in W_{v}$ such that $e\left(w, U_{1-i}\right) \geqslant \frac{\varepsilon}{4} n^{\alpha}$. Therefore, for every $v \in T_{0}$, Maker can choose a vertex $u \in V_{1} \backslash(R \cup T)$ such that $\{v, u\}$ is available and $e_{F}(w, R) \geqslant \frac{\varepsilon}{4} n^{\alpha}$ where $w$ is the vertex such that $\{u, w\} \in$ $P$. Maker repeats this procedure for every vertex in $v \in T_{0}$ and then move to the vertices of $T_{1}$. Thus, we can replace the vertices of $T_{0}$ in such a way that for every $v \in U_{0}$, $e_{F}\left(v, U_{1}\right) \geqslant \frac{\varepsilon}{4} n^{\alpha}$. Finally, Maker does the same for the vertices in $T_{1}$ and ensure that for every $v \in U_{1}, e_{F}\left(v, U_{0}\right) \geqslant \frac{\varepsilon}{4} n^{\alpha}$. This time, during the procedure, if a vertex $v \in T_{1}$ was replaced by $w$, then the degree of his neighbors in $U_{0}$ could become smaller than $\frac{\varepsilon}{4} n^{\alpha}$. But $\left|T_{1}\right| \leqslant n^{\alpha / 2}$ and therefore after replacing the vertices of $T_{1}$ we still have that for every 
$v \in U_{0}, e_{F}\left(v, U_{1}\right) \geqslant \frac{\varepsilon}{4} n^{\alpha}-n^{\alpha / 2}>\frac{\varepsilon}{5} n^{\alpha}$. Note that in every step Maker looks only at the neighbors of $v \in T$ that are not in the current $R$ and are not part of $T$. Therefore, by the end of this stage $\Delta\left(M_{2}\right) \leqslant 3$. This completes the proof for Stage II.

Stage III: First we show that after Stage II, the graph $F[R]$ contains a perfect matching $P_{1}$.

By the construction in Stage II and by Lemma 18, we have that the graph $G^{\prime}$ w.h.p. satisfies properties 1 and 2 of Claim 11 with $\left|A_{i}\right|=n^{\alpha}$. Therefore, w.h.p. $G^{\prime}$ contains a perfect matching $P_{1}$.

Finally, we show that in the next $n^{\alpha}$ rounds, w.h.p. Breaker will not claim any edge from $G^{\prime}$. Recall that by the end of the game there are at least $\frac{3}{4} \varepsilon n^{2}$ available edges in the graph. Thus, the probability for Breaker to claim an edge from $G^{\prime}$ in the next round is at most $\frac{4 n^{2 \alpha}}{3 \varepsilon n^{2}} \cdot b=(1-\varepsilon) \frac{4 n^{2 \alpha+1}}{3 \varepsilon n^{2}}$. Therefore, the probability for Breaker to claim an edge from $G^{\prime}$ in the next $n^{\alpha}$ rounds is at most $n^{\alpha} \cdot(1-\varepsilon) \frac{4 n^{2 \alpha+1}}{3 \varepsilon n^{2}}=(1-\varepsilon) \frac{4 n^{3 \alpha+1}}{3 \varepsilon n^{2}}=o(1)$ for $0<\alpha<\frac{1}{3}$. We thus choose $\alpha$ to be (for instance) $\frac{1}{4}$.

All in all, during stage III w.h.p. Breaker will not claim edges from $G^{\prime}$. Furthermore, from Claim 11, w.h.p. Maker can find a perfect matching $P_{1}$ in $G^{\prime}$ and thus claim all the edges of $P_{1}$ in the next $n^{\alpha}$ rounds.

This completes the proof of Theorem 2 .

\subsection{Random Breaker $k$-connectivity game}

In order to prove Theorem 3 we use the Random-Breaker Hamiltonicity game (Theorem 1) and the Random-Breaker perfect-matching game (Theorem 17). Since this game is also bias monotone, we can assume that $b=(1-\varepsilon) \frac{n}{k}$.

First we present a strategy for Maker and then we prove that w.h.p. Maker can follow this strategy.

Strategy $\boldsymbol{S}_{\boldsymbol{k}}$ : Maker (arbitrarily) partitions the vertices of $K_{n}$ into $k$ disjoint sets $V_{1}, \ldots, V_{k-1}, U$ where each $V_{i}$ has size $\left\lfloor\frac{n}{k-1}\right\rfloor$ and $U=V\left(K_{n}\right) \backslash\left(\bigcup V_{i}\right)$ (that is, $|U|<k-1$ ). For every $1 \leqslant i \leqslant k-1$ let $G_{i}=K_{n}\left[V_{i}\right]$ and for every $1 \leqslant i<j \leqslant k-1$ let $B_{i j}$ be the bipartite graph with $V\left(B_{i j}\right)=V_{i} \cup V_{j}$ and $E\left(B_{i j}\right)=\left\{\{u, v\} \mid v \in V_{i}, u \in V_{j}\right\}$.

Stage I: Maker plays the perfect-matching game on every $B_{i j}$ according to $S_{P M}$ with $\alpha=\frac{1}{4}$. Maker follows $S_{P M}$ on $E\left(B_{12}\right)$ to create a perfect matching in $B_{12}$, then on $E\left(B_{13}\right)$, etc. In total, Maker plays (sequentially) $\left(\begin{array}{c}k-1 \\ 2\end{array}\right)$ games on $\left(\begin{array}{c}k-1 \\ 2\end{array}\right)$ boards. If at some point Maker cannot follow $S_{P M}$ then he forfeits the game. Note that this stage takes $(1+o(1))\left\lfloor\frac{n}{k-1}\right\rfloor\left(\begin{array}{c}k-1 \\ 2\end{array}\right)$ rounds and by the end of this stage Maker's graph contains a perfect matching between any two sets $V_{i}, V_{j}$. Also, according to $S_{P M}, \Delta(M)=O(1)$.

Stage II: Maker plays the Hamiltonicity game on every $G_{i}$ according to $S_{H a m}$. Maker follows $S_{H a m}$ on $G_{1}$ to create a Hamilton cycle in $G_{1}$, then on $G_{2}$, etc. In total, Maker plays $k-1$ games on $k-1$ boards. If at some point Maker cannot follow $S_{\text {Ham }}$ then he forfeits the game. Note that this stage takes $\left(1+o(1)\left\lfloor\frac{n}{k-1}\right\rfloor(k-1)\right.$ rounds and by the end of this stage Maker has $k-1$ disjoint cycles each of length $\left\lfloor\frac{n}{k-1}\right\rfloor$. Also, according to $S_{H a m}$ and $S_{P M}, \Delta(M)=O\left(n^{1 / 4}\right)$. 
Stage III: For every $u \in U$ Maker claims $k$ distinct edges $\{u, w\}$ such that $w \in$ $V\left(K_{n}\right) \backslash U$. This stage takes at most $k^{2}$ rounds.

Observe that if Maker successfully follows the strategy, his graph (on $\bigcup V_{i}$ ) consists of $k-1$ cycles of size $\left\lfloor\frac{n}{k-1}\right\rfloor$ and between any two cycles there is a perfect matching. This graph is easily seen to be $k$-connected (see e.g., [8], Lemma 2.7). Adding $U$ to this graph and demanding that for every $u \in U, e\left(\{u\}, \cup V_{i}\right)=k$, we conclude that Maker's graph is indeed $k$-connected. In total, Maker plays at most $\left\lfloor\frac{n}{k-1}\right\rfloor(k-1)+\left\lfloor\frac{n}{k-1}\right\rfloor\left(\begin{array}{c}k-1 \\ 2\end{array}\right)+k^{2}+o(n)=$ $(1+o(1)) \frac{n k}{2}$ turns.

We show now that w.h.p. Maker can follow strategy $S_{k}$.

Stage I: First we show that the conditions of Lemma 18 holds. Indeed, observe that by the end of the game, Breaker has at most $\left(1-\frac{5}{6} \varepsilon\right)\left(\begin{array}{l}n \\ 2\end{array}\right)$ edges in the graph. Therefore, it follows from Lemma 16 that w.h.p. by the end of the game $\left|E_{B}\left(B_{i j}\right)\right|=e_{B}\left(V_{i}, V_{j}\right) \leqslant$ $\left(1-\frac{\varepsilon}{3}\right)\left(\left\lfloor\frac{n}{k-1}\right\rfloor\right)^{2}$ for every $1 \leqslant i<j \leqslant k-1$. Thus, the statement in Lemma 18 also holds for every graph $B_{i j}$ where $\frac{\varepsilon}{3} \leftarrow \frac{5}{6} \varepsilon$. Next we show, analogously to Lemma 20, that during the game, the degree in Breaker's graph in each $B_{i j}$ is not too large.

Lemma 21. Consider the (1:b) Maker-Breaker game on $E\left(K_{n}\right)$ where Breaker plays randomly and $b=(1-\varepsilon) \frac{n}{k}$. If after $(1+o(1)) \frac{k n}{2}$ turns of the game $\Delta(M)=O(1)$, then w.h.p. $\Delta\left(B \cap B_{i j}\right) \leqslant\left(1-\frac{\varepsilon}{2}\right)\left\lfloor\frac{n}{k-1}\right\rfloor$.

Proof. Let $v \in V$ and denote by $d_{B}(v)$ the degree of $v$ in $B\left[B_{i j}\right]$ by the end of the game. Then for $Z \sim H G\left(\left(\begin{array}{l}n \\ 2\end{array}\right)-O(n),(1-\varepsilon)\left(\begin{array}{c}n \\ 2\end{array}\right),\left\lfloor\frac{n}{k-1}\right\rfloor\right)$ and $\mu=\mathbb{E}(Z)$, using Lemma 8 we have,

$$
\begin{aligned}
\operatorname{Pr}\left[d_{B}(v)>\left(1-\frac{\varepsilon}{2}\right)\left\lfloor\frac{n}{k-1}\right\rfloor\right] & \leqslant(1+o(1)) \cdot \operatorname{Pr}\left[Z>\left(1-\frac{\varepsilon}{2}\right)\left\lfloor\frac{n}{k-1}\right\rfloor\right] \\
& \leqslant(1+o(1)) \cdot \operatorname{Pr}\left[Z>\left(1+\frac{\varepsilon}{4}\right) \mu\right\rfloor \\
& \leqslant(1+o(1)) e^{-\frac{\varepsilon^{2}}{48} \mu} \leqslant(1+o(1)) e^{-\frac{\varepsilon^{2}}{48 k}(1-2 \varepsilon) n} .
\end{aligned}
$$

Therefore, by the union bound,

$$
\operatorname{Pr}\left[\exists v \in B_{i j} \text {, s.t. } d_{B}(v)>\left(1-\frac{\varepsilon}{2}\right)\left\lfloor\frac{n}{k-1}\right\rfloor\right\rfloor \leqslant\left\lfloor\frac{n}{k-1}\right\rfloor(1+o(1)) e^{-\frac{\varepsilon^{2}}{48 k}(1-2 \varepsilon) n} .
$$

Finally,

$$
\begin{aligned}
\operatorname{Pr} & {\left[\exists B_{i j}, \exists v \in B_{i j}, \text { s.t. } d_{B}(v)>\left(1-\frac{\varepsilon}{2}\right)\left\lfloor\frac{n}{k-1}\right\rfloor\right] } \\
& \leqslant\left(\begin{array}{c}
k-1 \\
2
\end{array}\right) \cdot 2\left\lfloor\frac{n}{k-1}\right\rfloor \cdot(1+o(1)) e^{-\frac{\varepsilon^{2}}{48 k}(1-2 \varepsilon) n}=o(1) .
\end{aligned}
$$

All in all, according to the proof of Theorem 17 (and using the union bound on $\left(\begin{array}{c}k-1 \\ 2\end{array}\right)$ events), Maker can build w.h.p. a perfect matching in each $B_{i j}$ in $(1+o(1))\left\lfloor\frac{n}{k-1}\right\rfloor$ rounds. Therefore, to build $\left(\begin{array}{c}k-1 \\ 2\end{array}\right)$ perfect matchings Maker needs $(1+o(1))\left\lfloor\frac{n}{k-1}\right\rfloor\left(\begin{array}{c}k-1 \\ 2\end{array}\right)$ rounds. 
Stage II: In order to follow the strategy in this stage, we need to show that similarly to the proof of Theorem 1, also here there are many available edges between any two subsets of vertices of polynomial size. For this we use Lemma 16. Note that it follows from the strategy and the duration of the game that yet again w.h.p. $|E(M)|=o\left(n^{2}\right)$ and $|E(B)| \leqslant\left(1-\frac{5}{6} \varepsilon\right)\left(\begin{array}{l}n \\ 2\end{array}\right)$ and thus the statement of Lemma 16 holds here as well.

Next we show, similarly to Lemma 15, that during the game, the degree in Breaker's graph in each $G_{i}$ is not too large.

Lemma 22. Consider the $(1: b)$ Maker-Breaker game on $E\left(K_{n}\right)$ where Breaker plays randomly and $b=(1-\varepsilon) \frac{n}{k}$. If $\Delta(M)=O\left(n^{\delta}\right)$ (where $0<\delta<1$ is a constant), then w.h.p. $\Delta\left(B \cap G_{i}\right) \leqslant\left(1-\frac{\varepsilon}{2}\right)\left\lfloor\frac{n}{k-1}\right\rfloor$.

Proof. Let $v \in V$ and denote by $d_{B}(v)$ the degree of $v$ in $B\left[G_{i}\right]$ by the end of the game. Then for $Z \sim H G\left(\left(\begin{array}{c}n \\ 2\end{array}\right)-n^{\delta+1},(1-\varepsilon)\left(\begin{array}{c}n \\ 2\end{array}\right),\left\lfloor\frac{n}{k-1}-1\right\rfloor\right)$ and $\mu=\mathbb{E}(Z)$, using Lemma 8 we have,

$$
\begin{aligned}
\operatorname{Pr}\left[d_{B}(v)>\left(1-\frac{\varepsilon}{2}\right)\left\lfloor\frac{n}{k-1}\right\rfloor\right] & \leqslant(1+o(1)) \cdot \operatorname{Pr}\left[Z>\left(1-\frac{\varepsilon}{2}\right)\left\lfloor\frac{n}{k-1}\right\rfloor\right] \\
& \leqslant(1+o(1)) \cdot \operatorname{Pr}\left[Z>\left(1+\frac{\varepsilon}{4}\right) \mu\right\rfloor \\
& \leqslant(1+o(1)) e^{-\frac{\varepsilon^{2}}{48} \mu} \leqslant(1+o(1)) e^{-\frac{\varepsilon^{2}}{48 k}(1-2 \varepsilon) n} .
\end{aligned}
$$

Therefore, by union bound,

$$
\operatorname{Pr}\left[\exists v \in G_{i} \text {, s.t. } d_{B}(v)>\left(1-\frac{\varepsilon}{2}\right)\left\lfloor\frac{n}{k-1}\right\rfloor\right] \leqslant\left\lfloor\frac{n}{k-1}\right\rfloor \cdot(1+o(1)) e^{-\frac{\varepsilon^{2}}{48 k}(1-2 \varepsilon) n} .
$$

Finally,

$$
\begin{aligned}
\operatorname{Pr} & {\left[\exists G_{i}, \exists v \in G_{i}, \text { s.t. } d_{B}(v)>\left(1-\frac{\varepsilon}{2}\right)\left\lfloor\frac{n}{k-1}\right\rfloor\right\rfloor } \\
& \leqslant(k-1) \cdot\left\lfloor\frac{n}{k-1}\right\rfloor \cdot(1+o(1)) e^{-\frac{\varepsilon^{2}}{48 k}(1-2 \varepsilon) n}=o(1) .
\end{aligned}
$$

All in all, according to the proof of Theorem 1 (and using the union bound on $k-1$ events), w.h.p. Maker can build a Hamilton cycle in each $G_{i}$ in $(1+o(1))\left\lfloor\frac{n}{k-1}\right\rfloor$ rounds. Therefore, to build $k-1$ cycles, Maker needs $(1+o(1))\left\lfloor\frac{n}{k-1}\right\rfloor(k-1)$ rounds.

Stage III: From Lemma 20 we know that w.h.p. for every $u \in U$ we have $d_{B}(u) \leqslant$ $\left(1-\frac{\varepsilon}{2}\right) n$. But $|U|<k$ and therefore w.h.p. by the end of the game $e_{F}(\{u\}, V \backslash U)>k$ and Maker can claim w.h.p. $k$ neighbors of $u$ in $k$ rounds.

Taking it all together, w.h.p. Maker can follow the suggested strategy and build the desired graph. This completes the proof of Theorem 3. 


\section{Random Maker games}

In this section we consider the random-player setting where Maker plays randomly and claims in every round $m$ elements among all available elements, uniformly at random. Here we prove Theorems 4, 5 and 6 .

\subsection{Random Maker positive minimum degree game - Breaker's side}

In this section we prove Theorem 4 . We show that if $m=O(\ln \ln n)$ then w.h.p. Breaker can isolate a vertex in Maker's graph and thus typically win every game whose winning sets consist of spanning subgraphs with a positive minimum degree.

Proof. Let $\varepsilon>0$ be small enough constant and let $m \leqslant\left(\frac{1}{2}-\varepsilon\right) \ln \ln n$. Since claiming more edges can only help Maker, we can assume $m=\left(\frac{1}{2}-\varepsilon\right) \ln \ln n$. We show that Breaker has a strategy such that following it w.h.p. he can claim all $n-1$ edges incident to one vertex within $\frac{c n \ln n}{m}$ rounds of the game ( $c$ is a constant to be chosen later). Observe that after $\frac{c n \ln n}{m}$ rounds, there are only $\frac{c n \ln n}{m}(m+1)=(1+o(1)) c n \ln n$ claimed elements on the board of the game. Therefore, at any point during the game, there are at least $\left(\begin{array}{l}n \\ 2\end{array}\right)-\frac{c n \ln n}{m}(m+1)=(1-o(1)) \frac{n^{2}}{2}$ available elements on the board. We say that a vertex $v$ is free if $E_{M}(\{v\}, N(v))=\varnothing$. Breaker chooses a free vertex $v$ and tries to claim all the edges incident to $v$. If Maker claimed some edge $\{v, w\}$ before Breaker was able to claim all edges incident to $v$, then in the next round Breaker chooses another free vertex and repeats the procedure. If at some point during the first $\frac{c n \ln n}{m}$ rounds there is no free vertex in the graph, then Breaker forfeits the game. Every time Breaker moves to a new free vertex, we say that Breaker started a new attempt. Now we need to show that w.h.p. if Breaker follows his strategy then he will succeed in one of the attempts.

First we show that at any point during the first $\frac{c n \ln n}{m}$ rounds of the game, there exists a free vertex in the graph.

Claim 23. There exists a constant $c>0$ such that after $\frac{c n \ln n}{m}$ rounds of the game, w.h.p. there exists a vertex $v$ which is isolated in Maker's graph.

Proof. In the first $\frac{c n \ln n}{m}$ rounds, Maker claims $c n \ln n$ edges. The proof is similar to the Coupon Collector problem with minor changes. Assume that every time that Maker touches a free vertex, he actually touches two free vertices (otherwise it is only in favor of Breaker). Let $\tau_{i}$ be the number of turns until Maker touches $2 i$ different vertices and let $\tau$ be the number of turns until Maker touches all the vertices. Then $\tau=\tau_{1}+\left(\tau_{2}-\tau_{1}\right)+$ $\left(\tau_{3}-\tau_{2}\right)+\cdots+\left(\tau_{\frac{n}{2}}-\tau_{\frac{n}{2}-1}\right)$. Also, for every $i, \tau_{i+1}-\tau_{i}$ is stochastically dominated from above by $A_{i} \sim \operatorname{Geo}\left(\frac{2}{1-o(1)} \cdot \frac{n-2 i}{n}\right)$ and from below by $B_{i} \sim G e o\left(\frac{(n-2 i) n-c n \ln n}{n^{2}}\right)$ (the number of unclaimed edges from the $n-2 i$ free vertices divided by the number of available edges in the graph). Furthermore, since $B_{i}$ and $A_{i}$ are all independent random variables, we have that $\mathbb{E}(\tau) \geqslant C n \ln n$ (for some constant $C>0$ ) and $\operatorname{Var}(\tau)=\Theta\left(n^{2}\right)$. Then by Chebyshev we have that

$$
\operatorname{Pr}(|\tau-C n \ln n|>d n \ln \ln n) \leqslant \Theta\left(\frac{n^{2}}{d^{2} n^{2} \ln ^{2} \ln n}\right)=o(1) .
$$


Therefore, for a constant $c$ which satisfies $c n \ln n<C n \ln n-d n \ln \ln n$ we have that $\operatorname{Pr}(\tau<c n \ln n)=o(1)$.

Next, we show that the probability for Breaker to claim all edges from one vertex in one attempt is not too small.

Claim 24. The probability for Breaker to be able to claim in one attempt all edges incident to some free vertex $v$ is at least $\frac{1}{\ln ^{1-4 \varepsilon} n}$.

Proof. For some vertex $v$, the probability for Maker to touch this vertex at some turn is at most $\frac{n-1}{\left(\begin{array}{c}n \\ 2\end{array}\right)-\frac{c n \ln n}{m}(m+1)}=\frac{2}{n}(1+o(1))$. Therefore, the probability for Breaker to claim all edges from some vertex is at least $\left(1-\frac{2}{n}(1+o(1))\right)^{m(n-1)}$ (we want Maker to "fail" at least $m(n-1)$ times $)$. Note that,

$$
\left(1-\frac{2}{n}(1+o(1))\right)^{m(n-1)}=\left(1-\frac{2}{n}(1+o(1))\right)^{\left(\frac{1}{2}-\varepsilon\right)(n-1) \ln \ln n} \geqslant e^{-(1-4 \varepsilon) \ln \ln n},
$$

this completes the proof.

During the game, Breaker has at least $\frac{\frac{c n \ln n}{m}}{n}=\frac{c \ln n}{m}$ attempts. Let $X$ be the number of successful attempts, then,

$$
\operatorname{Pr}[X<1] \leqslant \operatorname{Pr}\left[\operatorname{Bin}\left(\frac{c \ln n}{m}, \frac{1}{\ln ^{1-4 \varepsilon} n}\right)=0\right]=\left(1-\frac{1}{\ln ^{1-4 \varepsilon} n}\right)^{\frac{c \ln n}{m}}=o(1) .
$$

Thus w.h.p. Breaker succeeds at least once and is able to claim all edges from some vertex $v$ in the first $\frac{c n \ln n}{m}$ rounds. This completes the proof.

\subsection{Random Maker Hamiltonicity game}

In this section we prove Theorem 5. One way to show that Maker's graph contains w.h.p. a Hamilton cycle, is to prove that Maker's graph is a good (connected) expander. In order to do so, we use the well-known Box game, firstly introduced by Chvátal and Erdős in [6].

Let $n$ and $s$ be integers. In the $(a: b)$-Box $(n \times s)$ game there are $n$ boxes, each with $s$ elements. In every round, BoxMaker claims $a$ elements from the boxes, and BoxBreaker claims $b$ elements from the boxes. BoxBreaker's goal is to claim at least one element from each box by the end of the game. After we analyze the game Box in our setting (and a variant called the $d$-Box game), we show that Maker's graph is indeed w.h.p. an expander and then we show that w.h.p. it contains a Hamilton cycle.

\subsubsection{Building an expander}

Lemma 25. Let $n$ be an integer, let $d$ and $\alpha$ be constants and let $A>9 d / \alpha$. Then for $b=A \ln \ln n$ w.h.p. random-BoxBreaker wins the $(1: b)$-random-BoxBreaker Box $(d n \times s)$ game assuming $s=\alpha n$. 
Proof. of Lemma 25 We show that w.h.p. BoxBreaker wins within $\Theta\left(\frac{n \ln n}{b}\right)$ rounds. Specifically, we argue that after $\frac{4 d n \ln n}{b}$ rounds, the elements claimed by BoxBreaker w.h.p. satisfy two properties that allow BoxBreaker to win the game.

Property 1: First we show that in the first $\frac{4 d n \ln n}{b}$ rounds, w.h.p. every box that BoxBreaker has not touched, has at least $\frac{s}{3}$ available elements. Let $A_{i}$ be the event "the $i^{\text {th }}$ box to reach $\frac{s}{3}$ elements of BoxMaker was not touched by BoxBreaker in the next $\frac{s}{3}$ rounds". Now, the probability for BoxBreaker to touch some box $i$ with his next element is at least $\frac{s / 3}{d n s}=\frac{1}{3 d n}$, Therefore,

$$
\operatorname{Pr}\left(A_{i}\right) \leqslant\left(1-\frac{1}{3 d n}\right)^{b s / 3} \leqslant e^{-\frac{1}{9 d n} b s}=e^{-\frac{\alpha}{9 d} A \ln \ln n}=\left(\frac{1}{\ln n}\right)^{\frac{\alpha}{9 d} A} .
$$

Note that the number of events $A_{i}$ that can occur is at most $\frac{4 d n \ln n / b}{s / 3}=\frac{12 d \ln n}{\alpha b}$. Thus, for $A>9 d / \alpha$ we have that

$$
\operatorname{Pr}\left(\exists i \in[n] \text {, s.t. } A_{i}\right) \leqslant \frac{12 d \ln n}{\alpha b} \cdot \operatorname{Pr}\left(A_{i}\right) \leqslant \frac{12 d \ln n}{\alpha A \ln \ln n} \cdot \frac{1}{(\ln n)^{\frac{\alpha}{9 d} A}}=o(1) .
$$

Property 2: Next we show that w.h.p. BoxBreaker touches every box that had at least $\frac{s}{3}$ available elements in the first $\frac{4 d n \ln n}{b}$ rounds. We say that a box $i$ is free if all the elements in $i$ are either available or belong to BoxMaker. The probability for BoxBreaker to touch the box $i$ with at least $\frac{s}{3}$ available elements in a given turn is at least $\frac{1}{3 d n}$. Therefore, for a given box, the probability that after the first $\frac{4 d n \ln n}{b}$ rounds the box has at least $\frac{s}{3}$ available elements and was not touched by BoxMaker is at most $\left(1-\frac{1}{3 d n}\right)^{\frac{4 d n \ln n}{b}}=o\left(\frac{1}{d n}\right)$. Using the union bound over all boxes we have that the probability that after the first $\frac{4 d n \ln n}{b}$ rounds there is a box that was not touched by BoxBreaker is $1-o(1)$.

All in all, the set of elements belong to BoxBreaker w.h.p. satisfies properties $1+2$ and thus after $\frac{4 d n \ln n}{b}$ rounds (that is $4 d n \ln n$ turns of BoxBreaker), w.h.p. BoxBreaker claimed at least one element from each box.

The following corollary is obtained from Lemma 25. In this corollary, we study a version of the Box game, the $d$-Box game, where $d$ is a positive integer. This game will be used later for proving Theorem 5 and Lemma 27. In the game $d$-Box $(n \times s)$ there are $n$ boxes, each with $s$ elements, and two players, $d$-Maker and $d$-Breaker. In the $(m: 1)$ random-Maker $d$-Box game, $d$-Maker plays randomly and claims in each round exactly $m$ previously unclaimed elements while $d$-Breaker claims exactly one element. The goal of $d$-Maker is to claim at least $d$ elements in each box.

Corollary 26. Let $\alpha, d, n>0$ be integers, let $A>9 d^{2} / \alpha$ be a constant and let $m=$ $A \ln \ln n$. Then w.h.p. d-Maker wins the $(m: 1)$-random-Maker d-Box $(n \times s)$ game where $s=\alpha n$. Furthermore, in every box there were at least $\frac{s}{2}$ available elements when $d$-Maker claimed $\frac{d}{2}$ elements from it. 
Proof. At the beginning of the game, $d$-Maker partitions each of the $n$ boxes into $d$ subboxes, each of size $\frac{s}{d}$. Then, for $s=\alpha n$ we have $d n$ boxes each of size $\frac{\alpha n}{d}$. From Lemma 25, playing the random-BoxBreaker $\operatorname{Box}\left(d n \times \frac{\alpha n}{d}\right)$ game, BoxBreaker w.h.p. wins when $b \geqslant A \ln \ln d n$ where $A>9 d^{2} / \alpha$. Therefore for $m \geqslant A \ln \ln n$ and $A>9 d^{2} / \alpha d$-Maker wins w.h.p. the $d$-Box game. Moreover, he typically does so within $\Theta\left(\frac{n \ln n}{m}\right)$ rounds of the game.

For the second part, we showed that w.h.p. $d$-Maker is the winner of the game against any strategy of $d$-Breaker. Now, assume by contradiction that at some point of the game there exists a box $i$ with at least $\frac{s}{2}-\frac{d}{2}+1$ elements of $d$-Breaker and at most $\frac{d}{2}-1$ elements of $d$-Maker. Then, by looking at $d$-Maker's partition of box $i$ into $d$ sub-boxes, $d$-Breaker could have claimed all the elements (but one) from $\frac{d}{2}-1$ sub-boxes and all the $\frac{s}{d}$ elements from another sub-box, thus winning the game, which is clearly a contradiction.

The next step in the proof of Theorem 5 (and also of Theorem 6) is to show that w.h.p. Maker's graph is an expander. For this we need the following lemma.

Lemma 27. For every positive integer $k$ there exist constants $\delta>0$ and $C_{1}>0$ for which the following holds. Suppose that $m^{\prime} \geqslant C_{1} \ln \ln n$. Then in the $\left(m^{\prime}: 1\right)$-random-Maker game played on the edge set of $K_{n}$, w.h.p. after $O\left(\frac{n \ln n}{m^{\prime}}\right)$ rounds, Maker's graph is an $(R, 2 k)$-expander, where $R=\delta n$.

Proof. Let $d=16 k$ and let $C_{1}>20 d^{2}$. At the beginning of the game, Maker assigns edges of $K_{n}$ to vertices so that each vertex gets about $\frac{n}{2}$ edges incident to it. To do so, let $D_{n}$ be any tournament on $n$ vertices such that for every vertex $v \in V,\left|N^{+}(v)\right|=\left|N^{-}(v)\right| \pm 1$ if $n$ is even and $\left|N^{+}(v)\right|=\left|N^{-}(v)\right|$ if $n$ is odd. For each vertex $v \in V\left(D_{n}\right)$, define $A_{v}=E\left(v, N^{+}(v)\right)$. Note that for every $v \in V\left(K_{n}\right)$ we have that $\left|A_{v}\right|=\left\lfloor\frac{n-1}{2}\right\rfloor$ or $\left|A_{v}\right|=\left\lceil\frac{n-1}{2}\right\rceil$ and that all the $A_{v}$ 's are pairwise disjoint. For the sake of simplicity, during the proof we sometimes omit floor and ceiling signs whenever these are not crucial.

Now, note that if a graph $G$ is an $(R, 2 k)$-expander, then $G \cup\{e\}$ is also an $(R, 2 k)$ expander for every edge $e \in E\left(K_{n}\right)$. Therefore, claiming extra edges can not harm Maker in his goal of creating an expander and we can assume $m^{\prime}=C_{1} n \ln n$ (if $m^{\prime}$ is larger then it is only in favor of Maker).

Our goal is to show that w.h.p. Maker's graph, after $\Theta(n \ln n)$ turns of the game, is an $(R, 2 k)$-expander. Here, we think of Maker as $d$-Maker in the game $d$-Box with appropriate parameters, where the boxes are $\left\{A_{v}: v \in V\left(K_{n}\right)\right\}$. Since $d$-Maker is w.h.p. the winner of the $d$-Box game, we will prove that Maker also wins this game.

Consider a game $d$-Box $(n \times s)$ for $d=16 k$ and $s=\frac{n-1}{2}$.

Since $\left|A_{v}\right|=\frac{n-1}{2}$, by Corollary 26 we have that for $m^{\prime}>20 d^{2} \ln \ln n$ w.h.p. $d$-Maker wins the $d$-Box game after at most $\Theta(n \ln n)$ turns of the game. Furthermore, for every box $A_{v}$, if Maker claimed so far less than $8 k$ elements from $A_{v}$, then there are at least $\frac{n}{4}$ available elements in the box. For some edge $e$ in Maker's graph, we say that $e=\{u, v\}$ was chosen by the vertex $v$ if $e \in A_{v}$.

According to the setting of the game, in every round Maker claims $C_{1} \ln \ln n$ available elements randomly. This can be viewed as follows. In every turn we can think of Maker 
as choosing first a vertex $v$ with an appropriate probability $\left(\frac{f(v, i)}{\sum_{u \in[n]} f(u, i)}\right.$, where $f(v, i)$ is the number of available elements in $A_{v}$ just before the $i^{\text {th }}$ turn of the game) and then choosing a random element from $A_{v}$, rather than choosing a random available edge from the graph.

We now prove that Maker's graph is w.h.p. an $(R, 2 k)$-expander. Let $M^{*}$ be Maker's graph after Maker, as $d$-Maker, wins the $d$-Box game. First, we look in a sub-graph of $M^{*}$ obtained in the following way. For every set $A_{v}$ we consider only the first $\frac{d}{2}$ elements claimed by $d$-Maker. Then, we look at the graph $L \subseteq M^{*}$ formed by these edges. It is evident that if $L$ is an $(R, 2 k)$-expander then $M^{*}$ is also an $(R, 2 k)$-expander. Therefore, it is enough to prove now that w.h.p. $L$ is an $(R, 2 k)$-expander. Suppose that the graph $L$ is not an $(R, 2 k)$-expander, then there is a vertex subset $A,|A|=a \leqslant R$, in the graph $L$, such that $N_{L}(A) \subseteq N$, where $|N|=2 k a-1$. Since Maker claimed from each $A_{v}$ at least $8 k$ edges and $k \geqslant 1$, we can assume that $a \geqslant 5$ and that there are at least $4 k a$ of Maker's edges incident to $A$, all of them chosen by vertices from $\mathrm{A}$ and all went into $A \cup N$. Assume that at some point during the game Maker chose an edge with one vertex $v \in A \cap A_{v}$ and whose second endpoint is in $A \cup N$. This means that there are at least $\frac{n}{4}$ available elements in the box $A_{v}$ and therefore at that point of the game, there are at least $\frac{n}{4}$ unclaimed edges incident to $v$. The probability that at that point Maker chose an edge at $v$ whose second endpoint belongs to $A \cup N$ is thus at most $\frac{|A \cup N|-1}{n / 4}$. It follows that the probability that there are at least $4 k a$ edges chosen by vertices of $A$ that end up in $A \cup N$ is at most $\left(\frac{(2 k+1) a-2}{n / 4}\right)^{4 k a}$. Thus, the probability that there are at least $4 k a$ edges between $A$ and $A \cup N$ is at most $\left(\frac{(2 k+1) a-2}{n / 4}\right)^{4 k a}<\left(\frac{12 k a}{n}\right)^{4 k a}$. Therefore the probability that there is such a pair of sets $A, N$ as above is at most

$$
\begin{aligned}
\sum_{a=5}^{R}\left(\begin{array}{l}
n \\
a
\end{array}\right)\left(\begin{array}{c}
n-a \\
2 k a-1
\end{array}\right)\left(\frac{12 k a}{n}\right)^{4 k a} & \leqslant \sum_{a=5}^{R}\left(\frac{n e}{a}\left(\frac{n e}{2 k a}\right)^{2 k}\left(\frac{12 k a}{n}\right)^{4 k}\right)^{a} \\
& =\sum_{a=5}^{R}\left(\frac{e^{2 k+1} k^{2 k} 12^{4 k}}{2^{2 k}}\left(\frac{a}{n}\right)^{2 k-1}\right)^{a}=o(1)
\end{aligned}
$$

The last equality is due to the fact that for $5 \leqslant a \leqslant \sqrt{n}$

$$
\left(\frac{e^{2 k+1} k^{2 k} 12^{4 k}}{2^{2 k}}\left(\frac{a}{n}\right)^{2 k-1}\right)^{a}=\left(\Theta\left(\frac{1}{n^{k-0.5}}\right)\right)^{5}=o\left(\frac{1}{n}\right),
$$


and for $\sqrt{n} \leqslant a \leqslant R$ with $R=\delta n$ where $\delta<(12 k e)^{-6}$ is a constant,

$$
\begin{aligned}
\left(\frac{e^{2 k+1} k^{2 k} 12^{4 k}}{2^{2 k}}\left(\frac{a}{n}\right)^{2 k-1}\right)^{a} & \leqslant\left(\frac{e^{2 k+1} k^{2 k} 12^{4 k}}{2^{2 k}}\left(\frac{R}{n}\right)^{2 k-1}\right)^{\sqrt{n}} \\
& =\left(\frac{e^{2 k+1} k^{2 k} 12^{4 k}}{2^{2 k}} \cdot \delta^{2 k-1}\right)^{\sqrt{n}} \\
& <\left(e^{2 k+1} k^{2 k} 12^{4 k} \cdot(12 k e)^{-12 k+6}\right)^{\sqrt{n}} \\
& =o(1 / n) .
\end{aligned}
$$

It follows that Maker is able to create an $(R, 2 k)$-expander w.h.p. in at most $\Theta\left(\frac{n \ln n}{m}\right)$ turns of the game.

Using Lemma 27, we now show that for $m \geqslant A n \ln n$, playing on the edge set of $K_{n}$, Maker's graph by the end of the random-Maker game contains w.h.p. a Hamilton cycle.

Proof of Theorem 5. Let $d=16$. Let $\delta=(13 e)^{-6}$ and let $A=21 d^{2}$.

We divide the game into three parts and in each part we show that w.h.p. Maker's graph satisfying some "good" properties:

Part $\mathbf{I}$ - the graph $\mathbf{M}$ is an expander: Using Lemma 27 for $k=1$ and $R=\delta n$, Maker's graph is w.h.p. an $R$-expander before both players claimed in total $\Theta(n \ln n)$ edges from the graph.

Part II - the graph $\mathrm{M}$ is a connected expander: Denote Maker's graph from Stage I by $M_{1}$. Then $M_{1}$ is w.h.p. an $R$-expander, and by Lemma 14 the size of every connected component of $M_{1}$ is w.h.p. at least $3 R$. It follows that there are at most $\frac{n}{3 R}=\frac{1}{3 \delta}=: C$ connected components in $M_{1}$. In the next $2 \ln n$ turns the game, Maker's graph will become a connected $R$-expander. Observe that there are at least $(3 R)^{2}=9 \delta^{2} n^{2}$ edges of $K_{n}$ between any two such components. Also, in the next $2 \ln n$ turns of the game, the number of edges claimed by both players (together with Stage I) is $\Theta(n \ln n)$ and therefore the number of available edges between any two connected components during this stage is at least $9 \delta^{2} n^{2}-\Theta(n \ln n)=\Theta\left(n^{2}\right)$. Denote by $E_{1}, \ldots, E_{\left(\begin{array}{c}C \\ 2\end{array}\right)}$ the sets of edges between each two connected components. Maker's goal now is to claim at least one edge from each such set of edges in the next $2 \ln n$ turns. Denote by $\tau$ the number of Maker's turns until he touches every set at least once. The probability of Maker to claim an available edge from some set $E_{j}$ is at least $\frac{9 \delta^{2} n^{2}-\Theta(n \ln n)}{n^{2}} \geqslant 8 \delta^{2}$ and therefore

$$
\operatorname{Pr}(\tau>\ln n) \leqslant \sum_{i=1}^{\left(\begin{array}{c}
C \\
2
\end{array}\right)}\left(1-8 \delta^{2}\right)^{\ln n} \leqslant\left(\begin{array}{l}
C \\
2
\end{array}\right) \cdot e^{-8 \delta^{2} \ln n}=o(1) .
$$

Thus, in the next $2 \ln n$ turns of the game, Maker w.h.p. is able to claim at least one edge from each set and to make his graph connected. We denote the new graph Maker created by $M_{2}$. It is evident that $M_{2}$ is still an $R$-expander. 
Part III - completing a Hamilton cycle: If $M_{2}$ contains a Hamilton cycle, then we are done. Otherwise, by Lemma $13, E\left(K_{n}\right) \backslash M_{2}$ contains at least $\frac{(R+1)^{2}}{2}$ boosters. Observe that after adding a booster, the current graph is still an $R$-expander and therefore also contains at least $\frac{(R+1)^{2}}{2}$ boosters. Clearly, after adding at most $n$ boosters, $M_{2}$ becomes Hamiltonian. We show now that in this stage w.h.p., Maker reaches his goal after $\Theta(n)$ turns of the game.

In the next $\frac{4 n}{\delta^{2}}$ turns of Maker, he claims a random unclaimed edge from the graph. We are now looking for the probability for such edge to be a booster. There are at most $\frac{n^{2}}{2}-\frac{n}{2}$ unclaimed edges, and according to Lemma 13 there are at least $\frac{(\delta n+1)^{2}}{2}$ boosters in $M_{2}$. Since by the end of the game Breaker claimed at most $\Theta(n \ln n)$ edges, the number of available boosters after each turn of Maker at any point of this stage is at least $\frac{(\delta n+1)^{2}}{2}-|E(B)|=\frac{(\delta n+1)^{2}}{2}-\Theta(n \ln n)>(\delta n / 2)^{2}$. Therefore, in each turn of Maker, until he was able to claim $n$ boosters, the probability for Maker to claim a booster is at least

$$
\frac{(\delta n / 2)^{2}}{\left(\begin{array}{l}
n \\
2
\end{array}\right)} \geqslant \frac{\delta^{2}}{3} .
$$

Let $Y$ be the number of boosters Maker claimed in $\frac{4 n}{\delta^{2}}$ turns. Then by Lemma 7 ,

$$
\operatorname{Pr}(Y<n) \leqslant \operatorname{Pr}\left(\operatorname{Bin}\left(\frac{4 n}{\delta^{2}}, \frac{\delta^{2}}{3}\right)<n\right) \leqslant e^{-\left(\frac{1}{4}\right)^{2} \frac{4}{3} n}=o(1) .
$$

Thus in the next $\frac{4 n}{\delta^{2}}$ turns of Maker he is able w.h.p. to claim at least $n$ boosters. All in all, in the next $\Theta(n \ln n)$ turns of the game, Maker was able (w.h.p.) to claim $n$ boosters and thus Maker's graph in Hamiltonian.

\subsection{Random-Maker $k$-connectivity game}

In this section we prove Theorem 6 . In order to do so, we first prove that w.h.p. also in this game Maker's graph is a good expander (by using Lemma 27) and then show that in $n \ln n$ further turns it becomes $k$-connected.

Proof. Let $d=16 k, \delta=(13 k e)^{-6}$ and let $A=\max \left\{21 d^{2}, \frac{9-6 \ln \delta}{\delta}\right\}$. We divide the game into two parts and in each part we show that w.h.p. Maker's graphs satisfy some "good" properties:

Part I: Using Lemma 27 for $R=\delta n$, Maker's graph is w.h.p. an $(R, 2 k)$-expander before both players claimed in total $\Theta(n \ln n)$ edges in the graph.

Part II: Maker makes his graph a $\left(\frac{n+k}{4 k}, 2 k\right)$-expander in $n \ln n$ further turns of the game. During the next $n \ln n$ turns of the game, Maker played more than $A n$ turns for $A \geqslant \frac{9-6 \ln \delta}{\delta}$. It remains to prove that if Maker claims $A n$ edges randomly, then w.h.p. Maker's graph is a $\left(\frac{n+k}{4 k}, 2 k\right)$-expander. It is enough to prove that $E_{M}(U, W) \neq \varnothing$ for every two subsets $U, W \subseteq V$, such that $|U|=|W|=R$. Indeed, if there exists a subset $X \subseteq V$ of size $R \leqslant|X| \leqslant \frac{n+k}{4 k}$ such that $\left|X \cup N_{M}(X)\right|<(2 k+1)|X|$, then there are two subsets $U \subseteq X$ and $W \subseteq V \backslash\left(X \cup N_{M}(X)\right)$ such that $|U|=|W|=R$ and $E_{M}(U, W)=\varnothing$. 
We now prove that w.h.p., $E_{M}(U, W) \neq \varnothing$ for every $|U|=|W|=R$ after $A n$ turns of Maker. Let $U, W$ be two subsets such that $|U|=|W|=R$. Recall that in the entire game, both players claim at most $\Theta(n \ln n)$ edges. Thus the number of available edges between $U$ and $W$ at any point throughout this stage is at least $|U||W|-\Theta(n \ln n)>\frac{\delta^{2} n^{2}}{3}$ for a large $n$. Then the probability that Maker claims an edge $e \in E(U, W) \backslash E_{B}(U, W)$ is at least $\frac{\delta^{2} n^{2} / 3}{\left(\begin{array}{c}n \\ 2\end{array}\right)} \geqslant \frac{\delta^{2}}{3}$. So at the end of Stage II,

$$
\operatorname{Pr}\left(E_{M}(U, W)=\varnothing\right)=\operatorname{Pr}\left(E(U, W) \backslash E_{B}(U, W)=\varnothing\right) \leqslant\left(1-\frac{\delta^{2}}{3}\right)^{A n} .
$$

Using the union bound, we get that the probability that there exist two subsets $U, W$, $|U|=|W|=R$ such that $E_{M}(U, W)=\varnothing$ is at most

$$
\begin{aligned}
\left(\begin{array}{c}
n \\
\delta n
\end{array}\right)\left(\begin{array}{c}
n \\
\delta n
\end{array}\right)\left(1-\frac{\delta^{2}}{3}\right)^{A n} & \leqslant\left(\frac{e n}{\delta n}\right)^{\delta n}\left(\frac{e n}{\delta n}\right)^{\delta n}\left(1-\frac{\delta^{2}}{3}\right)^{A n} \\
& \leqslant\left(\frac{e}{\delta}\right)^{2 \delta n}\left(1-\frac{\delta^{2}}{3}\right)^{A n} \\
& \leqslant e^{2 \delta n-2 \delta n \ln \delta-\delta^{2} A n / 3} \\
& =o(1) .
\end{aligned}
$$

Then w.h.p. by Lemma 12 , since $\left(\frac{n+k}{4 k}\right) \cdot 2 k \geqslant \frac{1}{2}(|V|+k)$, Maker's graph is $k$-connected and he wins the game.

\section{Concluding remarks and open problems}

(1) In this paper we studied the random-player Maker-Breaker games such as the Hamiltonicity game, the perfect-matching game and the $k$-vertex-connectivity game. In the random-Breaker version, we were able to give asymptotically tight results for the value of the critical bias of the games. We actually proved that the critical bias for these random-Breaker games is asymptotically the maximal value of $b$ that allows Maker's graph to satisfy the desired property. Namely, we proved that for every $\varepsilon>0$ if $b \leqslant(1-\varepsilon) \frac{n}{2}$ then Maker typically wins the random-Breaker Hamiltonicity game, if $b \leqslant(1-\varepsilon) n$ then Maker typically wins the random-Breaker perfect-matching game and if $b \leqslant(1-\varepsilon) \frac{n}{k}$ then Maker typically wins the random-Breaker $k$-connectivity game. However, in the random-Maker version, we were only able to determine the correct order of magnitude for the critical bias $m^{*}$. In the random-Maker versions of the above games we proved that $m^{*}=\Theta(\ln \ln n)$, that is, the maximal value of $m$ that allows Breaker to be the typical winner of the game is of order $\ln \ln n$. One can try to find the exact multiplicative constant in this term.

(2) Note that we only considered random-player Maker-Breaker games played on $E\left(K_{n}\right)$, and there is still nothing known about random-player games played on different 
boards. Thus, it would be interesting to study the critical bias for random-player Maker-Breaker games played on different boards, for example, games played on the edge set of some general graph $G$, games played on the edge set of a random graph, games played on the edge set of a hypergraph, etc.

(3) With a bit more careful implementation of the same arguments one can also take $\varepsilon=\varepsilon(n)$ to be a concrete vanishing function of $n$, but we decided not to pursue this goal here.

\section{Acknowledgements}

The authors would like to thank the referees of the paper for their careful reading and many helpful remarks.

\section{References}

[1] N. Alon and J. H. Spencer, The Probabilistic Method, $3^{\text {rd }}$ ed. Wiley, New York, 2008.

[2] B. Bollobás, Random graphs, $2^{\text {nd }}$ ed. Cambridge University Press, 2001.

[3] J. Beck, Combinatorial Games: Tic-Tac-Toe Theory, Cambridge University Press, 2008.

[4] M. Bednarska and T. Euczak, Biased positional games for which random strategies are nearly optimal, Combinatorica 20 (2000), 477-488.

[5] S. Ben-Shimon, A. Ferber, D. Hefetz and M. Krivelevich, Hitting time results for Maker-Breaker games, Random Structures Algorithms 41 (2012), 23-46.

[6] V. Chvátal and P. Erdős, Biased positional games, Annals of Discrete Math. 2 (1978), 221-228.

[7] D. Clemens, A. Ferber, R. Glebov, D. Hefetz and A. Liebenau, Building spanning trees quickly in Maker-Breaker games, SIAM J. Discrete Math., to appear.

[8] D. Clemens, A. Ferber, M. Krivelevich and A. Liebenau, Fast strategies in MakerBreaker games played on random boards, Combin. Probab. Comput. 21 (2012), 897915.

[9] A. Ferber and D. Hefetz, Weak and strong $k$-connectivity game. European J. Combin. 35 (2014), 169-183.

[10] A. Ferber, M. Krivelevich and G. Kronenberg, Efficient winning strategies in randomturn Maker-Breaker games. submitted. arXiv:1408.5684.

[11] H. Gebauer and T. Szabó, Asymptotic random graph intuition for the biased connectivity game, Random Structures Algorithms 35 (2009), 431-443.

[12] D. Hefetz, M. Krivelevich, M. Stojaković, and T. Szabó, Fast winning strategies in Maker-Breaker games. J. Combin. Theory Series B, 99 (2009), 39-47.

[13] D. Hefetz, M. Krivelevich, M. Stojaković and T. Szabó, Positional Games (Oberwolfach Seminars), Birkhäuser, 2014. 
[14] S. Janson, T. Łuczak and A. Ruciński, Random graphs, Wiley, 2000.

[15] M. Krivelevich, The critical bias for the Hamiltonicity game is $(1+o(1)) n / \ln n, J$. American Math. Soc. 24 (2011), 125-131.

[16] A. Lehman, A solution of the Shannon switching game, J. Soc. Industrial Appl. Math., 12 (1964), 687-725.

[17] Y. Peres, O. Schramm, S. Sheffield and D. B. Wilson, Random-turn hex and other selection games, American Math. Monthly, 114 (2007), 373-387.

[18] D. B. West, Introduction to Graph Theory, $2^{\text {nd }}$ ed. Prentice Hall, 2001. 